UCRL-53946

Distribution Category UC-701

UCRL- -53946

DE91 010045

\title{
NUSTART:
}

\section{A PC Code for \\ Nuclear Structure and Radiative Transition Analysis and Suplementation}

Users' Guide

G. L. Larsen

D. G. Gardner

M. A. Gardner

Manuscript date: October 1990

\section{LAWRENCE LIVERMORE NATIONAL LABORATORY University of California - Livermore, California - 94551}

Available to DOE and DOE contractors from the Office of Scientific and Technical Information

P.O. Box 62, Oak Ridge, TN 37831 Prices available from (615) 576-9601, FT5 626-8401

Available from: National Technical Information Service - US. Department of Commerce 5285 Port Royal Road - Springfield, VA 22161 - A04 - (Microfiche A01?

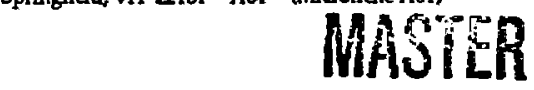




\section{Contents}

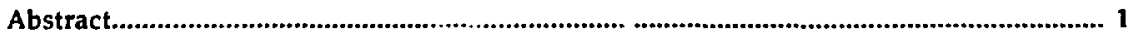

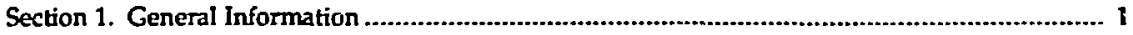

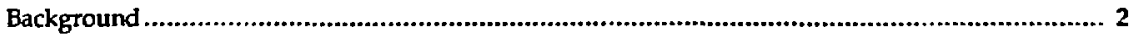

System Requirements ................................................................................................ 2

The NUSTART Code..................................................................................................... 2

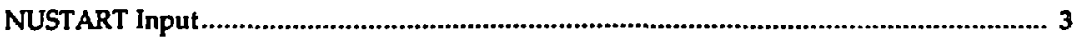

NUSTART Modes .................................................................................................. 7

Using the NUSTART Code.................................................................................... 10

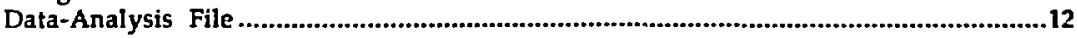

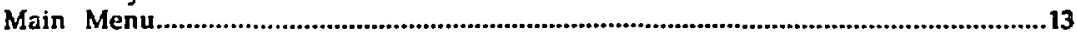

Section 2. Data File Error Analysis Mode .........................................................................15

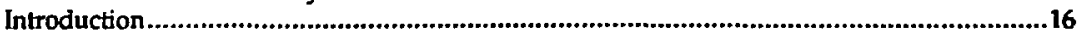

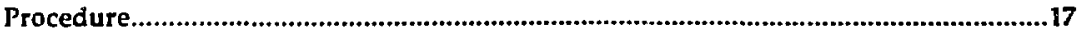

Section 3. Interactive Dala File Generation Mode.............................................................19

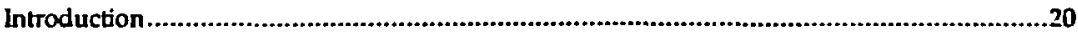

Procedure......................................................................................................22

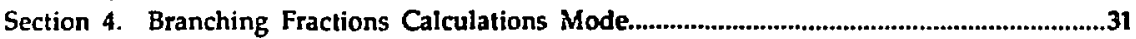

Introduction......................................................................................................................32

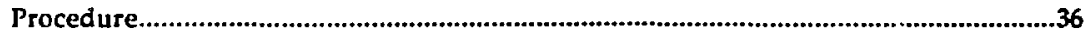

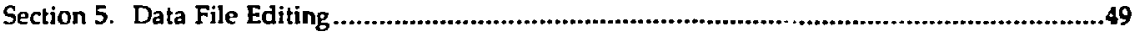

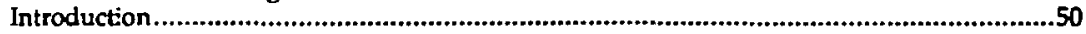

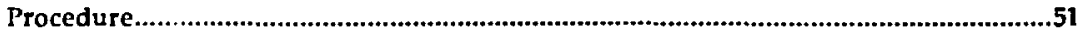

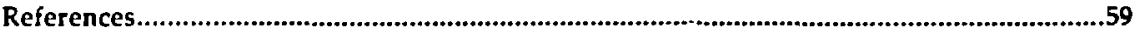

Appendices .....................................................................................................................61

A. Energy-Dependent E1 Multipole Strength Function.....................................................61

B. Sample NUSTART Input and Output Files............................................................63

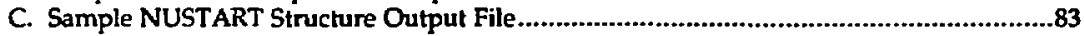

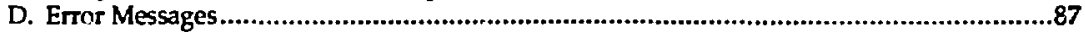




\title{
A PC Code for Nuclear Structure and $\underline{\text { Radiative Transition }}$ Analysis and Supplementation
}

\author{
Users' Guide
}

\begin{abstract}
NUSTART is a computer program for the IBM PC/AT. It is designed for use with the nuclear reaction cross-section code STAPLUS, which is a STAPRE-based CRAY computer code.that is being developed at the Lawrence Livermore National Laboratory. The NUSTART code was developed to handle large sets of discrete nuclear levels and the multipole transitions among these levels; it operates in three modes. The Data File Error Analysis mode analyzes an existing STAPLUS input file containing the levels and their multipole transition branches for a number of physics and/or typographical errors. The Interactive Data File Generation mode allows the user to create input files of discrete levels and their branching fractions in the format required by STAPLUS, even though the user enters the information in the (different) format used by many people in the nuclear structure field. In the Branching Fractions Calculations mode, the discrete nuclear level set is read, and the multipole transitions among the levels are computed under one of two possible assumptions: (1) the levels have no collective character, or (2) the levels are all rotational band heads. Only E1, M1, and E2 transitions are considered, and the respective strength functions may be constants or, in the case of E1 transitions, the strength function may be energy dependent. The first option is used for nuclei near ciosed shells; the bandhead option may be used to vary the E1, M1, and E2 strengths for interband transitions. $\mathrm{K}$-quantum number selection rules may be invoked if desired.
\end{abstract}

\section{Section 1. General Information}

NUSTART is a computer program for the IBM PC/AT. It is designed for use with the nuclear reaction cross-section code STAPLUS, which is a STAPRE-based CRAY computer code.that is being developed at the Lawrence Livermore National Laboratory. The NUSTART code was developed to handle large sets of discrete nuclear levels and t 2 multipole transitions among these levels; it operates in three modes. The Data File Error Analysis mode analyzes an existing STAPLUS input file containing the levels and their multipole transition branches for a number of physics and/or typographical errors. The Interactioe Dala File Generation mode allows the user to create input files of discrete levels and their branching fractions in the format required by STAPLUS, even though the user enters the information in the (different) format used by many people in the nuclear structure field. In the Branching Fractions Calculations mode, the discrete nuclear level set is read, and the multipole transitions among 
the levels are computed under one of two possible assumptions: (1) the levels have no collective character, or (2) the levels are all rotational band heads. Only E1, M1, and E2 transitions are considered, and the respective strength functions may be constants or, in the case of $E 1$ transitions, the strength function may be energy dependent. The first option is used for nuclei near closed shells; the bandhead option may be used to vary the E1, Mi, and E2 strengths for interband transitions. K-quantum number selection rules may be invoked if desired.

\section{Background}

The accuracy with which one may calcuiate nuciear reaction cross sections with the modern coces now in use, particularly codes of the Hauser-Feshbach plus precompound, particleevaporation type, depends upon the quality and accuracy of a wide variety of input information. Indeed, these modern computer codes may not produce results as dependable as those from the older, less sophisticated codes because the modem codes require large amounts of physics information specific to the problem at hand and rely less on global parameterizations. Limited inpu: information, not to mention input errors, can easily produce misleading and unacceptable results from the modern codes.

An important example of some of the required input is that of large sets of discrete nuclear levels and the nultipole transitions among thern. Accurate and extensive level sets and their decay branches, are extremely imporlant for the calculation of nuclear-reaction cross sections for excitation energies up to a few tens of $\mathrm{MeV}$; they are indispensable if isomer populations are required. In addition to their use in caiculating particle and photon cross sections and energy spectra, the discicte level sets provide much needed low-energy physics information. The closeness of the parity fraction of the level set to 0.5 is one indicator of how well the discrete levels will match the continuum level density. Also, a preponderance of one parity may signal possible problems with an isomer population calculation, since M1 and $E 2$ transitions among the levels may be unduly emphasized. The spin cut-off parameler, which governs the angularmomentum distribution as a function of energy, may be computed from the discrete levels and then compared with energy-dependent theoretical expressions. The actual spin distribution of the discrete levels may be fitted, in the least-squares sense, to theoretical expressions, and the spin cut-off parameter extracted in that way also. Comparison of the level density and its variation with energy, as obtained from the level sets, with that obtained from continuum level density expressions can often be useful.

\section{System Requirements}

NUSTART runs on IBM PC/AT and AT-compatible machines with 640K of memory. It was written using the Microsoft C Compiler, Microsoft Corp., Bellevue, Washington, and the utility routines from the C UTILITY LIBRARY, Essential Software Inc., Maplewood, New Jersey. It was was built on a system using a color monitor with an Enhanced Graphics Adapter (EGA) board; although NUSTART may run on monochrome systems or on a system using Coior Graphics Adapter (CGA) or Video Graphics Array (VGA) boards, it has not been tested on such systems. The system must have a 5-1/4-in., high-density floppy disk drive.

\section{The NUSTART Code}

NUSTART is designed for use with the nuclear reaction cross-section code STAPLUS, which is a STAPRE-based ${ }^{1}$ CRAY computer code in development at the Lawrence Livermore National Laboratory. NUSTART was first written to check for typographical and other errors in our large sets of discrete levels and their multipole transitions. ${ }^{2}$ Some of the sets contain over 900 discrete levels, each of which may decay via up to 30 multipole branches. Some of these 
level sets come directly from structure-code calculations, such as for permanent rotors ${ }^{3}$ or Interacting Boson Approximation (IBA) calculations. ${ }^{4}$ In some of these rases, the modeled discrete-level information can be checked internally by the structure codes themselves. However, many of our level sets are assembled "by hand" from a variety of experimental and theoretical sources. Usually, it is virtually impossible to proof these sels of levels and branches manually.

The NUSTART code was developed to handle these large sets of discrete nuclear levels and the multipole transitions among these levels. It operates in three modes: the Data File Error Analysis mode, the Interactioe Data File Generation mode, and the Branching Fractions Calculations mode.

In th:is report, we show the input formats for nuclear structure information and for nuclear level sets and their decay functions and provide step-by-step instructions for using each mode. In appendices, we derive the energy-dependent E1 multipole strength function, provide sample NUSTART input and output files, and explain error messages.

\section{NUSTART Input}

\section{Format for Nuclear Structure Information}

The nuclear structure information must be provided in the Fortran format specified by

STAPLUS. The nuclear level set consists of a header card, followed by the levels themselves.

Card 1 (13, A9, 13, A65)

\section{Column}

\section{$1-3$}

4-12

13-15

$16-80$

NDISK
NAME

\section{Content}

Number of discrete levels Name of nucleus (e.g., IR193) Blank Comment

\section{Cards 2 (6(F6.3, F4.1, 12))}

\section{Column}

$\begin{array}{ll}1-6 & \text { ED(1) } \\ 7-10 & \text { SD(1) } \\ 11-12 & \text { KPD(1) } \\ 13-18 & \text { ED(2) } \\ 19-22 & \text { SD(2) } \\ 23-24 & \text { KPD(2) } \\ & \text { etc. }\end{array}$

\section{Content}

Energy of ground state (MeV) Spin of ground state Parity of ground state $(+1$ or -1$)$ Energy of first excited state (MeV) Spin of first excited state Parity of first excited state

Six levels per card, up to NDISK levels; maximum value of NDISK is 999. 
The multipole transitions among the discrete levels are supplied as branching fractions that sum to unity for each radiating level. Cards for the ground state and any isomeric levels are omitted.

Cards 3 (13, A3, 12, 2X, 10F7.3)

\section{Column}

$1-3$

4-6

$7-8$

9-10

11-17

18-24

\section{Content}

LEVEL Numiver of the radiating level, (e.g., 2 for first excited state).

ENDS If ENDS = END, this signals the end of this set of multipole branching information.

NBR

Number of multipole branches from this level, $\leq 30$. If $N B R>10$, another card is read. Three cards ( 30 branches per radiating level) are maximum. If NBR is blank, only one card is read (number of branches $\leq 10$ ).

Blank

TVZD(1) TVZD(i) are the encoded branching TVZD(2) fractions.

etc.

The branching fractions, TVZD(i), are encoded as floating point numbers: III $X X X$, starting in column 11. Here, $X X X$ is the branching fraction to final level, III. Use 111.000 for a branching fraction of unity (100\%). The 1 in card in the set should be blank except for END in columns 4-6. Table 1 shows an example. 
Table 1. Example of the format required for the nuclear level sets and their decay branching fractions. $a, b$

\begin{tabular}{|c|c|c|c|c|c|c|c|c|c|c|c|}
\hline \multicolumn{12}{|c|}{54 IR191 } \\
\hline 0.000 & $1.5+1$ & 0.082 & $0.5+1$ & 0.129 & $2.5+1$ & 0.171 & $5.5-1$ & 0.179 & $1.5+1$ & 0.342 & $3.5+1$ \\
\hline 0.351 & $2.5+1$ & 0.391 & $3.5-1$ & 0.503 & $4.5+1$ & 0.504 & $3.5+1$ & 0.539 & $1.5+1$ & 0.557 & $6.5-1$ \\
\hline 0.589 & $2.5+1$ & 0.591 & $7.5-1$ & 0.625 & $0.5+1$ & 0.650 & $1.5-1$ & 0.654 & $4.5-1$ & 0.686 & $3.5+1$ \\
\hline 0.748 & $2.5+1$ & 0.763 & $1.5+1$ & 0.787 & $3.5+1$ & 0.800 & $2.5-1$ & 0.812 & $4.5+1$ & 0.832 & $5.5+1$ \\
\hline 0.875 & $4.5+1$ & 0.878 & $4.5-1$ & 0.902 & $2.5-1$ & 0.919 & $5.5-1$ & 0.963 & $7.5-1$ & 0.977 & $3.5-1$ \\
\hline 0.987 & $4.5+1$ & 0.991 & $5.5+1$ & 1.004 & $6.5+1$ & 1.037 & $8.5-1$ & 1.050 & $5.5+1$ & 1.092 & $6.5-1$ \\
\hline 1.106 & $5.5+1$ & 1.127 & $9.5-1$ & 1.136 & $5.5-1$ & 1.179 & $3.5-1$ & 1.180 & $0.5-1$ & 1.211 & $1.5-1$ \\
\hline 1.230 & $5.5-1$ & 1.233 & $5.5+1$ & 1.253 & $6.5-1$ & 1.270 & $4.5+1$ & 1.379 & $6.5+1$ & 1.397 & $6.5+1$ \\
\hline 1.418 & $7.5+1$ & 1.422 & $8.5-1$ & 1.424 & $2.5-1$ & 1.496 & $6.5+1$ & 1.601 & $8.5+1$ & 1.694 & $7.5+1$ \\
\hline & 2 & 1 & 1.0 & & & & & & & & \\
\hline & 3 & 1 & 1.0 & & & & & & & & \\
\hline & 5 & 2 & 1.24 & 2.76 & & & & & & & \\
\hline & 6 & 2 & 1.59 & 3.41 & & & & & & & \\
\hline & 7 & 3 & 1.49 & 3.02 & & & & & & & \\
\hline & 8 & 1 & 4.0 & & & & & & & & \\
\hline 9 & 9 & 2 & 3.59 & 6.41 & & & & & & & \\
\hline 10 & & 3 & 3.48 & 5.31 & & & & & & & \\
\hline 11 & & 4 & 1.45 & 2.11 & & & 5.19 & & & & \\
\hline 12 & & 1 & 4.0 & & & & & & & & \\
\hline 13 & & 3 & 1.74 & 3.24 & & & & & & & \\
\hline 14 & & 1 & 4.0 & & & & & & & & \\
\hline 15 & & 5 & 1.72 & 2.19 & & & 5.03 & 11.03 & & & \\
\hline 16 & & 4 & 1.02 & 2.12 & & & 8.80 & & & & \\
\hline 17 & & 2 & 4.10 & 8.90 & & & & & & & \\
\hline 18 & & 3 & 1.20 & 3.40 & & & & & & & \\
\hline 19 & & 6 & 1.02 & 3.04 & & & 6.05 & 7.05 & 11. & & \\
\hline 20 & & 3 & 3.01 & 5.06 & 11 & & & & & & \\
\hline 21 & & 3 & 3.74 & 6.24 & & & & & & & \\
\hline 22 & & 2 & 8.56 & 17.44 & & & & & & & \\
\hline 23 & & 3 & 6.18 & 7.24 & 10 & & & & & & \\
\hline $2 d$ & & 2 & 6.23 & 9.77 & & & & & & & \\
\hline 25 & & 3 & 3.20 & 6.40 & & & & & & & \\
\hline 26 & & 2 & 6.10 & 9.90 & & & & & & & \\
\hline 27 & & 2 & 1.50 & 3.50 & & & & & & & \\
\hline 28 & & 2 & 8.15 & 17.85 & & & & & & & \\
\hline 29 & & 1 & 12.0 & & & & & & & & \\
\hline 30 & & 2 & 16.10 & 22.90 & & & & & & & \\
\hline 31 & & 3 & 6.74 & 9.24 & 24. & & & & & & \\
\hline 32 & & 3 & 9.33 & 10.60 & 23. & & & & & & \\
\hline 33 & & 2 & 9.98 & 24.02 & & & & & & & \\
\hline 34 & & 2 & 12.10 & 14.90 & & & & & & & \\
\hline 35 & & 5 & 6.15 & 9.15 & 18. & & 23.10 & 24.07 & & & \\
\hline 36 & & 1 & 26.0 & & & & & & & & \\
\hline 37 & & 4 & 6.20 & 9.35 & 18. & & 24.35 & & & & \\
\hline 38 & & 1 & 14.0 & & & & & & & & \\
\hline 39 & & 3 & 8.16 & 17.45 & 28. & & & & & & \\
\hline 40 & & 1 & 27.0 & & & & & & & & \\
\hline 41 & & 1 & 27.0 & & & & & & & & \\
\hline
\end{tabular}


Table 1. (Continued).

\begin{tabular}{lrrrrr}
\hline 42 & 1 & 41.0 & & & \\
43 & 2 & 26.83 & 36.17 & & \\
44 & 3 & 9.74 & 24.24 & 33.02 & \\
45 & 2 & 17.73 & 28.27 & & \\
46 & 4 & 3.05 & 9.41 & 19.22 & 35.32 \\
47 & 4 & 9.20 & 24.35 & 25.10 & 33.35 \\
48 & 3 & 23.51 & 24.22 & 32.27 & \\
49 & 2 & 24.23 & 33.77 & & \\
50 & 2 & 12.28 & 29.72 & & \\
51 & 1 & 40.0 & & & \\
52 & 3 & 24.74 & 33.24 & 49.02 & \\
53 & 2 & 33.96 & 49.04 & & \\
54 & 4 & 24.20 & 33.35 & 37.10 & 49.35 \\
END & & & & & \\
\hline
\end{tabular}

Branching fraction ards 1, 2, and 4 are omilted because LEVEL 1 is the ground state, and LEVELa 2 and 6 are isomers.

b This example does nol show TVZD(i) for i $>10$. 


\section{NUSTART Modes}

\section{Data File Error Analysis Mode}

In this mode, the NUSTART code examines a set of levels and branches (such as shown in Table 1) for a number of typographical and/or physics errors. Later in this manual, we will enumerate the types of errors subject to diagnosis. The code will write an analysis table similar to Table 2, which actually represents only a portion of the table produced when a level set for the nucleus ${ }^{193}$ Ir was analyzed. For illustrative purposes, the spin of level 4 was mistyped as 8.5 instead of 2.5 , and the multipole decay of level 4 was deliberately incorrectly assigned to level 3 instead of level 1. 
Table 2. A portion of the analysis table for ${ }^{193} \mathrm{Ir}$ that contains intentional input errors.

\section{GAMMA-RAY TRANSITIONS AMONG DISCRETE LEVELS}

Initial Level

N E J P
Final Level

N E J F EG BR F

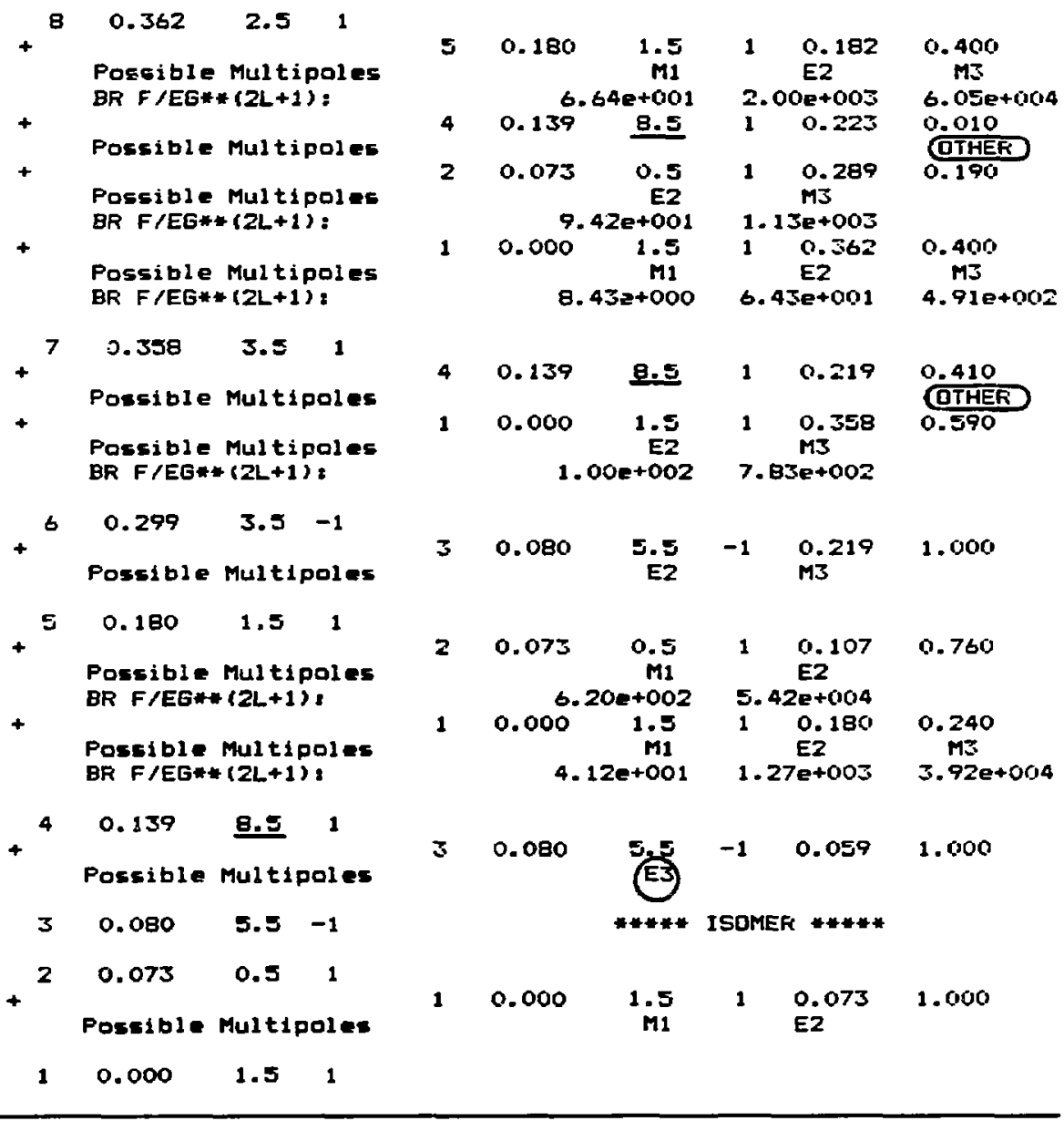


The following notation is used in Table 2: $N$ (level number), $E$ (level energy in MeV), J (level spin), P (level parity), EG (transition energy in MeV), and BR F (multipole branching fractions). Because the STAPLUS code considers multipole transitions with $\ell \leq 3$, higher multipole transitions are labeled "other," and a warning is issued to the PC monitor screen as well as being written (as a warning message) at the end of the analysis table. For our purposes, we consider mosat transiticns with $\ell>3$ to be indicative of isomeric states; thenefore, the warning usually represents an input error. In addition to indicaling the possible multipole type (M1, E2, etc.) that could give rise to the transition, the analysis table prints, if desired, the value of the branching fraction divided by transition energy raised to the $(2 f+1)$ power. If more than one decay branch is present, this information can be related either to relative gamma-ray strength functions or, if several transitions proceed to members of the same rotational band in a rigid rotor, to the Clebsch-Gordon coefficients.

\section{Interactive Data File Generation Mode}

Our format for the multipole branching fractions is not in common use. More often, one encounters tables of level energies, spins, and parities, along with the energies of the lower levels and the percentage of the total decay that populates a lower level. To facilitate the conversion of the nuclear structure and decay information to the format required by STAPLLS, a second mode called Interactive Dasa File Generation was added to NUSTART. Format descriptions and options are stated in a subsequent section. While the structure information is being converted to the format in Table 2, some error analyses are made, and the user is given the opportunity to correct the errors. When the level set is completed, the constructed STAPLUS file can be examined in the Error Aralysis mode to produce an output table like that in Table 1. One or more level sets may be constructed sequentially in this way, each with a header card containing up to 65 characters of descriplive information.

\section{Branching Fractions Calculations Mode}

In this third mode of NUSTART, the set of discrete levels minus the branching information is read, and the branching fractions are constructed under one of two possible assumptions: either the levels have no collective character, or the levels are all rotationa! bandheads wilh the remaining tand members deleted. The strengths of the multipole transitions among the levels are calculated using a relative value for each $\ell$-type of transition, multiplied by the transition energy raised to the $2 \ell+1$ power. Only E1, M1, and E2 transitions are permitted. The relative values for the strength functions may be calculated by NUSTART from the Weisskopf assumptions, they may either all be different constants supplied by the user or, in the case of E1 transitions, the E1 strength function may be made energy dependent as in a giant resonance treatment. If the levels are all collective bandheads, the user has the option of using the K-quantum number restrictions. Then, the K-quantum numbers must be supplied when $K$ does not equal J for that level. Also, up to six states may be specified in advance as isomers. The newly constructed STAPLUS input file is then run through the Data File Error Aralysis mode. and the output table is written.

In the case of permanent rotors, we assume that the intraband transitions are much faster than the interband transitions and that the decay from a band occurs only from the bandhead. Therefore, when the bandhead option is chosen, it is usually for one of the following reasons:

- To test the effect of the modification of the relative multipole strengths of the interband transitions among the discrele levels on a STAPLUS calculation of the population of an isomer.

- To add the possibility of E2 transitions. This would be appropriate when the initial nuclear structure code computed only dipole-type interband transitions.

Because only the bandhead levels are used in the bandhead option, the numbering of the initial and final levels will not correspond to the true level set that includes all band members. In a recent calculation involving $176_{\mathrm{Lu}}$, there were 291 levels up to $1.497 \mathrm{MeV}$, of which 62 were bandheads. When the bandhead option was invoked, NUSTART numbered the bandheads as levels with numbers 1 through 62 , and the branching frac:ion calculation identified the parent 
and daughter levels accordingly. In the complete set encompassing all band members, the trute bandhead level numbers ranged up to 285 . Thus, bandhead option level number 62 had to be changed to the true level number 285, bandhead option level number 61 had to be changex to true level number 272, etc. An option in NUSTART makes these shanges automatically. The user must supply a table consisting of pairs of bandhead option and true level numbers, either as an input file or interactively via the PC keyboard. The renumbering will not appcar in the data-analysis output file; however, the file created as input to STAPLUS will appear as in Table 1-with the addition of the branching fraction information repeated with the true numbering. At this time, the user must himself replace the original bandhead decay cards with the new cards. A possible future modification of NUSTART would have the code read in the complete set of branches and would replace the original bandhead decay cards with the new branch cards.

\begin{abstract}
* NOTE**
By mid-December 1989, a version of NUSTART had been run with a variety of level sets and with most of the option combinations listed in this users' guide. Should you notice any hitherto undetected errors, please iniorm us so that we can make corrections. Direct questions conceming the construction and/or design of the code to G. L. Larsen. Direct question. concerning the bisic physics and the approximations incorporated into the code to either D. G. Gardner or M. A. Gardner. We can be reached at the University of Califorsia, Lawrence Livermore National Laboratory, Nuclear Chemisiry Division, L-234, P. O. Box 808, Livermore, CA 94551, USA.
\end{abstract}

\title{
Using the NUSTART Code
}

Although the NUSTART program requires some understanding of the physics involved to make effective use of the program, it can be used with minimal computer expertise. With the exception of data or file-name entry, all commands are entered via the function keys fl through f10 and the ESC key, and all cursor control is done using the arrow keys on the key pad. Dataentry and file-name information are entered by filling out simple forms as they appear on the screen. In all cases, the form displayed on the screen shows what keys to use and what they do. These forms will ask you to type in the pertinent information. If there is more than one item on the form, NUSTART will automatically step you through the form as you fill out each item; alternatively, you can move to any item you wish by using the arrow keys to move the cursor.

\section{Data Entry Termination}

When you enter data into the computer through the keyboard, you must lot the computer know when you have finished by typing some delimiter as a data terminator. In this manual, we show which delimiter to use by putting it in parentheses at the end of the dataentry instruction. For example, the instruction:

\section{type in filename (Enter)}

would maan that you are to type in the name of a disk file with its path and terminate the entry of the name with the Enter key.

\section{Special Keys}

Always, the form displayed on the screen shows what keys to use and what they do. Certain keys, however, perform special functicns. 
Toggle Switches. Several forms use a toggle switch, usually the fl key, to toggle between a yes/no, 0/1, or on/off response. Pressing the toggle switch will cause the current, displayed setting to be toggled to the opposite setting.

Press f1C tu Continue. On most forms and menus, if you do not want to make any changes, bul simply want to go cn to the next step in the program, pressing the f10 key will gencrally accomplish this feat. The f 10 key also frequently acts as a terminator, letting the program know that you are through with that form.

ESC Key. The ESC key is a special key that is used differently in different situations; however, it always acts as some sort of "get nit" key. It may be used to instantly terminate a process without completing the process or, if a number of forms must be filled out to set up and start a process, it allows you to step back through the forms to make corrections.

Arrow Keys. The arrow keys ame used when control is needed to move a highlight bar or the cursor.

\section{Input Files}

The standard input file for the NUSTART code is the same as the letel dala input for the STAPLUS code. See Table 1 for the Fortran format required for this input file.

\section{Batch Files}

A batch file is a file containing multiple STAPLUS level input data sets concatenated together. Each data set is terminated with an END starting in column four of the last card of the data set, as in the normal STAPLUS level data set.

$$
\text { * * REMINDER * }
$$

NDISK is the variable name for the original number of discrete lesels in a level sel. This variable name is referred to frequently in this guide. 


\section{Data-Analysis File}

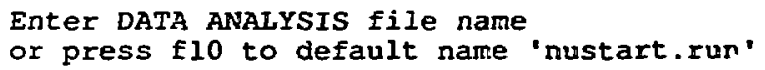

When execuled, the first thing NUSTART does is create a data-analysis file that automatically logs the following information:

- Start date and time.

- Input file name.

- The number of levels for the data set.

- The data-sel identification (id) card.

- A listing of the energy, spin, and parity for each level.

- Error messages.

- When the Data File Error Analysis mode is selected, the analysis information, inclurling a table of the multipole transitions and branching ratios, is saved in this $\log$ file.

You may either type in the name for the data-analysis file

\section{FILENAME (Enter)}

or use the default name "nustart.run" by pressing the flo key.

The data-analysis file is a cumulative file; that is, the code looks to see if the requested file already exists. If it does, the code opens the file and appends the new data to the end of the existing file. If the requested file does not cxist, a new file is created, and the data are written to it. 


\section{Main Menu}

\begin{tabular}{|l|}
\hline Select Operating Mode \\
\hline $\mathrm{f} 1=$ Data File Error Analysis \\
$\mathrm{f} 2=$ Interactive Data File Generation \\
$\mathrm{f} 3=$ Branching Fractions Calculations \\
$\mathrm{f} 4=$ Data File Editing \\
$\mathrm{ESC}=$ Terminate
\end{tabular}

This, the main menu for NUSTART, allows access to the four main functions of the program. You select the option you want by pressing the function key (f] - f4) sorresponding to that option. To end the program, you press the ESC key; this closes all files and gives a normal termination. If you end the program in any other way, i.e., (Ctr))C or (Ctri)Break, any open files will not be closed, and the data in the those files will be lost. Thus, if you do not care to save any information, using (Ctrl)C or (Ctrl)Break will kill the program at any point. 


\section{Section 2. Data File Error Analysis Mode}

\begin{tabular}{|l|} 
Select Operating Mode \\
\hline $\mathrm{f} 1=$ Data File Error Analysis \\
$\mathrm{f} 2=$ Interactive Data File Generation \\
$\mathrm{f} 3=$ Branching Fractions Calculations \\
$\mathrm{f} 4=$ Data File Editing \\
ESC $=$ Terminate
\end{tabular}

Press f1 to select the Data File Error Analysis mode. 


\section{Introduction}

In this mode of operation, an existing STAPLUS input file, such as shown in Table 1, is analyzed for severai types of errors. Then, a data-analysis table, illustrated in Table 2, is written, along with certain error messages (if any). As prewiously mentioned, data sels may be stacked, with a final blank card to signal the end of the stack.

NUSTART verifies the number of discrele levels in a set is by determining if the energy of the last level is $\leq 0.000$ or if the energy of the (NDISK +1 ) level is $>0.000$. If too few or too many levels are in the set, appropriate wamings are issued. Next, NUSTART checks the energies of the sequence of levels to see if any are out of (increasing) order.

The branching fraction cards are then read and checked to ensure that (1) there is no decay from level 1 (the ground state) and (2) that there are no radiating levels with level numbers that erceed NDISK. It in initial level decays $100 \%$ to one final level with level number $N$. the encoded branching is $N .000$. If a branch is found where the fraction $>0.000$, but $N \leq 0$, an error message is issued. Beginning with the highest energy level. NUSTART determines from the branching fraction card(s) for that initial radiating level the type and, based on the initial and final spins and parities, the multipolarity of each indicated transition to a lower level. The branching fractions are normalized in unity, if necessary. Only E1, M1, E2, M2, E3, and M3 transitions are identified and written into the data-analysis table. Higher multipolarity transitions are labeled "other," arid a warning message is issued. If desired, the branching fraction divided by the transition energy raised to the $(2 \ell+1)$ power is printed $(\ell$ is the multipolarity).

The user is cautioned that NUSTART does not examine the input file for all mnceivable errors. The code anticipates that the input will be in the STAPLUS general format, and this file is read in an nearly format-free mode.

\section{* WARNING **}

Some errors will be accepted by NUSTART, which wil! then create a data-analysis table that may or may not be correct. These same enors would be delected by STAPLUS, since it has a formatled read. Two examples will illustraic the situation:

- Assume that the level-description format is (F6.3, F4.1, 12). for the level energy, spin, and parity, respectively. If the spin is entered as 7;0 instead of 7.0, the unformatted read in NUSTART will reject the incorrect semicolon character and the following zero, and will understand the spin to be 7 . In this example, no incorrect conclusions will appear in the data-analysis table; however, should the spin value be half integer, such as 7.5, the error analysis will produce nonsense.

- A more dangerous error would be to indicate the level's parity as $=1$ instead of +1 or -1 . In this situation, NUSTART could reject the equals sign and find the parity to be +1 , and a reasonable-appearing but incorrect error analysis would be generated if the tue parity value happened $\mathrm{\omega}$ be -1 .

A final word of caution might be appropriate. It is quite possible for the data-analysis table file to be too large either to write onto a 5-1/4-in., high-density floppy disk or to be opened with a text editor. In our work, we upload this large file onto a main-frame computer to be viewed or to be sent to some output device. There are two ways to reduce the size of the dataanalysis table: request a printout for fewer than the actual number of levels, and/or suppress the printing of the branching fraction divided by the transition energy raised to the $(2 \boldsymbol{\ell}+1)$ power. These options will be mentioned again under the Output Print Options menu. 


\section{Procedure}

Enter input file name

Type in the name of the input file using the following format:

$$
\text { [disk:][pUh]file_name.ext(Enter) }
$$

The disk and path are needed only if the file does not exist on the current disk and is not in the current directory. If the program carinot find the tile, it gives a weming message and asks for the name again. Retype the name or press the ESC key to netum to the "Seloct Operating Mode" menu.

NUSTART reads the input file and nus an analysis on the den. If an error is encountered, NUSTART sends an error message to the terminal, wriles a more deailed error message to the data-malysis file, and quits processing the input file. NUSTART reburns to the min menu; there, you can either edit the input file with the Edit option or teminate NUST.ART and use you favorite lext editor to correct the data.

When the analysis is complete, two tables are written to the data-enalysis file: a table of the input data and branching fractions and a table of the multipole transitions. 


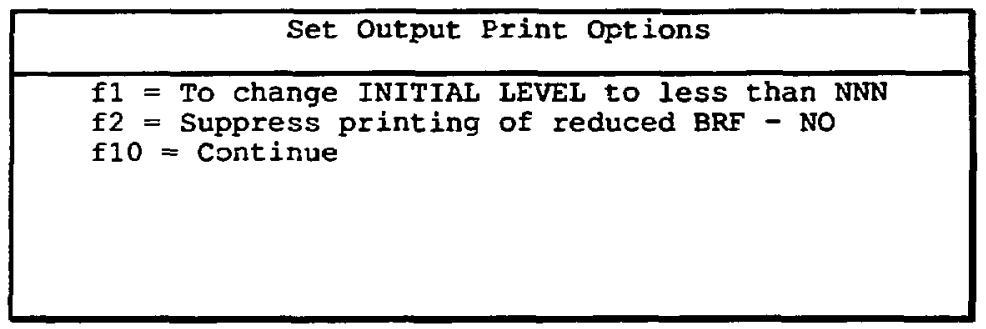

Once the analysis is complete, you can set the print options for the output

f1: Changes INITTAL LEVEL to less than NNN

$$
\text { ** NOTE *** }
$$

NNN is replaced by the actual number of levels in the file when the form is displayed.

You may now set the number of braxching levels that will be printed. As shown when the form comes up (NDISK), the default is to print all of the levels. Press $\mathrm{f}$ and type in the number of levels to print. If you do not want the data-analysis table written oul, use 0 (zero) as the initial level.

When you press $\mathrm{fl}$, the following change occurs in the menu:

\begin{tabular}{|l|}
\hline Set Output Print Options \\
\hline $\mathrm{f} 1=$ To change INITIAL LEVEL to less than NNN \\
$\mathrm{f} 2=$ Suppress printing of reduced BRE $-\mathrm{NJ}$ \\
$\mathrm{f} 10=$ Continue \\
Enter new maximum level number
\end{tabular}

Type in the maximum (INITIAL) level (Enter). A level of 0 (zero) will suppress the printing of the data-analysis table.

f2: Suppresses printing of reduced BRF - NO.

f2: Toggles a switch that allows you to control the printing of the branching function (BRF) information. The default is to print everything, except when only one branch occurs.

f10: Continues on with the next step.

The data-analysis file will be written, and you will be returned to the main menu for the next option. 


\section{Section 3. Interactive Data File Generation Mode}

\begin{tabular}{|l|}
\hline Select Operating Mode \\
\hline $\mathrm{fl}=$ Data File Error Anaiysis \\
$\mathrm{f} 2=$ Interactive Data File Generation \\
$\mathrm{f} 3=$ Branching Fractions Calculations \\
$\mathrm{f} 4=$ Data File Editing \\
$\mathrm{ESC}=$ Terminate
\end{tabular}

Press 2 for the Interactive Data File Generation mode. 


\section{Introduction}

Much of the nuclear-structure information that we receive comes in the form of manually constructed, typed tables rather than as disk files. As an example, we show in Table 3 the structure information for the first 12 levels in ${ }^{191} \mathrm{Ir}$, taken from Table 1.

Table 3. Nuclear structure information for the first few levels of 191 Ir.

\begin{tabular}{ccl}
\hline $\begin{array}{c}\mathrm{E} \\
(\mathrm{keV})\end{array}$ & $2]^{\pi}$ & \multicolumn{1}{c}{$\begin{array}{l}\text { Branching fractions/comments } \\
\text { [Daughter level energy (\% branching)] }\end{array}$} \\
\hline 0 & $3+$ & \multicolumn{1}{c}{ Stable } \\
82 & $1+$ & $0(100)$ \\
129 & $5+$ & $0(100)$ \\
171 & $11-$ & $0(100)$ \\
179 & $3+$ & $0(24), 82(76)$ \\
342 & $7+$ & $0(59), 129(41)$ \\
351 & $5+$ & $0(49), 129(2), 179(49)$ \\
391 & $7-$ & $171(100)$ \\
503 & $9+$ & $129(59), 342(41)$ \\
504 & $7+$ & $129(48), 179(31), 351(21)$ \\
539 & $3+$ & $0(45), 82(11), 129(25), 179(19)$ \\
557 & $13-$ & $171(100)$
\end{tabular}

In this example, the level energies are in units of $\mathrm{keV}$, the level spin is given as $2 \mathrm{~J}$ followed by the parity ( + or - ), and the multipole branching to lower levels is encoded as the daughter level energy followed by the percent branching in parentheses. Often a comment is appended, such as " $t_{1 / 2}=4.9 \mathrm{~s}$, isomer" for the fourth level. This allows the user to decide if the given half-life of a level is long enough for the level to be considered a long-lived isomer that doesn't decay in the time frame of a given problem. Often we consider levels with half-lives longer than about $10 \mathrm{~ns}$ as "stable"!

For our convenience, we require that the level energies be in units of either $\mathrm{keV}$ or $\mathrm{MeV}$, that the spins be listed either as J or 2], and that there be no more than 30 branches per radiating level. The percent branchings from a given level should sum to 100 , although NUSTART will perform this normalization if necessary. The levels should be listed in order of increasing energy, energies should be rounded to the nearest keV, and several levels may have the same energy, although the latter should be avoided by changing one or more level energies by a $\mathrm{keV}$ or so. A problem arises with transitions from higher-energy states. In the STAPLUS format, the daughter level is designated by its level number rather than by its energy. Hence, having several levels with the same energy creates no problem, as long as the user is careful in choosing the correct daughter level when it is fed from above. A maximum of 999 levels and their branches may be entered in this mode of NUSTART.

The STAPLUS input file that is being created in this Interactive mode is examined for certain errors as each ?evel and its branches are entered. If the level energies are not in 
increasing order, or if a transition is indicated to a level whose energy is not among the previous levels, NUSTART issues a waming, and the user is allowed to make a correction. At any time, the user may review the previous input and make changes. We have decided against permitting the easy insertion or deletion of a level card aind its branches in the previously construcled input, because NUSTART will not automatically renumber the daughter levels properly in the encoded STAPLUS file. The user should display the last correct input card (NDISK is automatically properly decremented) and recreate the subsequent cards anew. 


\section{Procedure}

Enter nucleus

Press $f 10$ to continue

Press ESC to go back to previous menu

Type in the name of the nucleus (Enter).

Enter: Acts as a terminator for the inpul and steps to the next form.

Press $\mathbf{f 0}$ to continue.

f10: Steps to the next form without changing any current settings that may exist.

Press ESC to go back to the previous menu.

\section{* * REMTNDER}

When crealing a file, you may enter the energy in terms of either keV or $\mathrm{MeV}$ and the spin in terms of either $\mathrm{J}$ or $2 \mathrm{~J}$. When the data are written to the output files, the STAPLUS file is always written in $\mathrm{MeV}$ and $\mathrm{J}$, whereas the structure file is written in $\mathrm{keV}$ and the selected spin (J or 2J). 
Piess fl to toggle

energy is in keV GFF

energy is in $\mathrm{MeV}$ ON

Press flo to continue

Press ESC to go back to previous menu

Press fl key to toggle spin.

f1: Acts as a toggle switch and lets you redefine the energy in units of keV or MeV.

Press f10 to continue.

f10: Steps to the next form.

Press ESC to go back to the previous menu.

Press fl key to toggle spin

$\operatorname{Spin}=\mathrm{J}$

Press fio to continue

Press ESC to go back to previous menu

Press fl to toggle spin.

f1: Acts as a toggle switch and lets you toggle the spin between $\mathrm{J}$ and $2 \mathrm{~J}$.

Press flo to continue.

f10: Steps to the next form.

Press ESC to go back to the previous menu. 
Enter comment

Press 110 to continue

Press ESC to go back to previous menu

Type in any comment you want put in the output file on card 1 . The comment may have a maximum of 45 characters.

Press Enter to terminate the comment entry and to step to the next form.

Press f10 to continue.

f10: Steps to the next form without causing any change in any current setting.

Press ESC to go back to the previous menu.

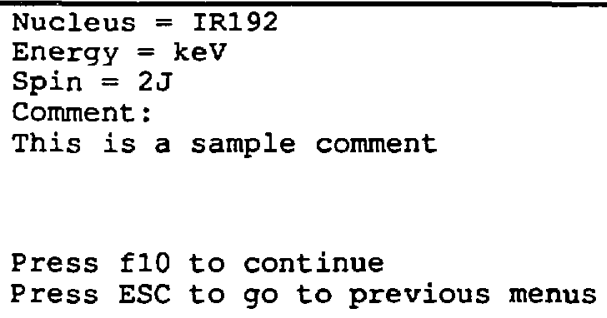

When all: the initial parameters have been set, they are displayed. You can reset any of them by using the ESC key to step back through the forms. Pressing f10 will move you on to the next step.

The filled-in form above is an example of a typical display. The user has set the nucleus to IR192, selected keV for the energy units, set a spin of 2], and added the comment "This is a sample comment." 


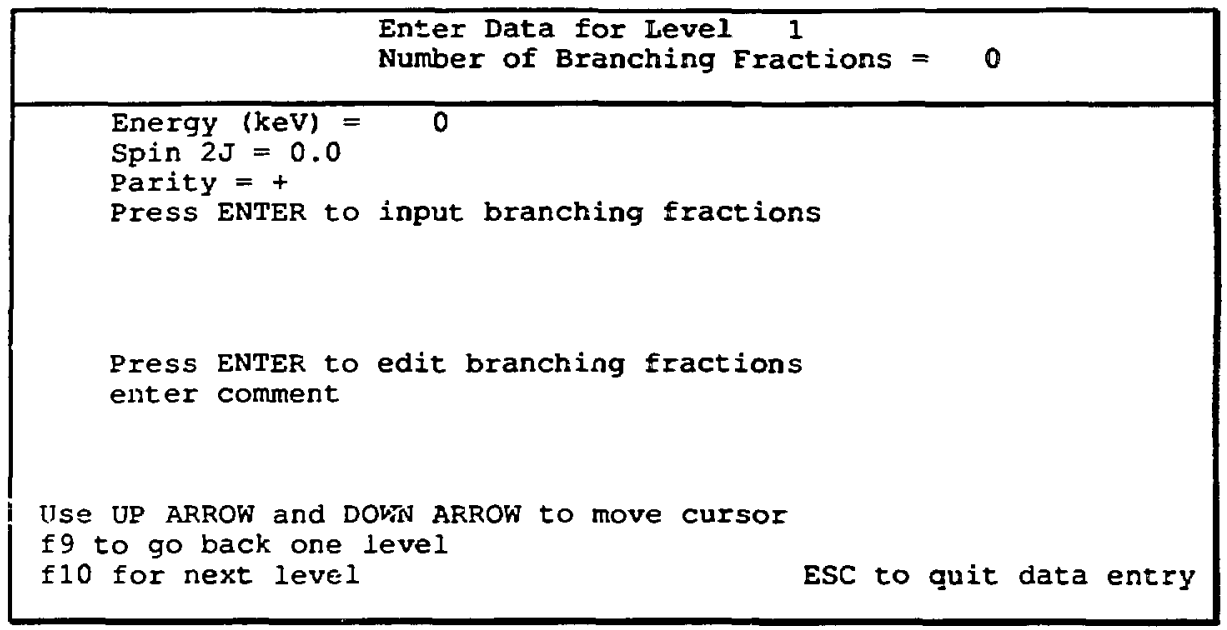

This is the main form for generating new data files. The first line shows the current level number. The second line shows the current number of branching fractions for the level.

As you fill in the form, the active line is shown by a highlight bar. When you fill in a line, the highight moves down to the next entry. You can also move up and down through the form by using the UP and DOWN arTow keys to move the highlight bar.

Energy $(\mathrm{keV})=0$.

To enter the energy, ype in the value (Er.ter) in keV.

Spin 2J =0.0.

To set the spin, type in the spin value (Enter).

Parity $=+$.

The parity is set by using ine 11 key as a toggle switch.

Press Enter to input branching fractions as percentages.

To input the branching percentages, press Enter. A new form will pop up to allow you to type in the branching percentage values (see below). 
Press Enter to edit branching percentages.

If any of the branches are incorrect, you may edit them by bringing up the editing form. The editing form shows the branches in columns of energy level and percent of decay. Ensure that the branching percentages sum to 100 .

Enter comment.

To put a comment in the output file for the current level, type in the comment (Enter). This comment will be written after the branching ratios. The comment may have a maximum of 45 characters.

Press $f 9$ to go back one level.

f9: Moves you down to the previous level. You may change any value in this level.

\section{- WARNING *.}

The code uses the current level as the highest level when the input is ended. Therefore, if you have used the $19 \mathrm{kcy}$ to move down through the levels, be certain to relum to the bight:: level before ending.

Press f10 for next level.

f10: Moves you to the next highest level.

Press ESC to quit data entry.

When you have filled in the data for all the levels wanted, press the ESC key to quit. 
When you select to input branching fractions, the following form is brought up:

Enter Branching Percentages

(as blank delimited pairs)

A maximum of 30 branching percentages may be entered

To enter the branching percentages, type them in as blonk delimited pairs in the form:

$$
\text { kevel_energy } \phi_{\text {_decay }} \text { level_energy } \%_{0} \text { decay... }
$$

The level energy is entered in the units (keV or MeV) set at the onset of the editing session. The percent decay may be entered either as integer numbers or as decimal values (c.g., 33, 33.3, .03, etc.). When all the branching percentages are in, press $\mathrm{fl} 0 \mathrm{w}$ quil and retum. You may. at any time, nullify the data entry and retum to the calling form by pressing the ESC key.

$$
\text { ** NOTE }
$$

The maximum value for the energy is $9995 \mathrm{keV}$. The percent of decay can have a maximum of four chareters (e.g. 33.3. 22.5. etc.): any value greater than 100 percent will be treated as 100 . 
When you select to edit the branching fractions, the following form is brought up:

\section{Branching percentages for level NNN}

EL \& EL $\quad$ EL

Use the cursor arrows to move cursor to value to be changed f1 to change value

flo to return

Use the arrow keys to move the cursor to the value to be changed. You may move through the branching percentages at will, and change them in any order.

\section{-. NOTE *..}

NNN is replesed by the actual level number when the form is displayed.

Press $\mathrm{fl}$ to open the value for change.

Type in the new value (Enter).

Press $\mathrm{f} 10$ to end the editing and to retum to the data entry form.

You may cancel the editing session at any time and nullify all changes made by pressing the ESC key. 


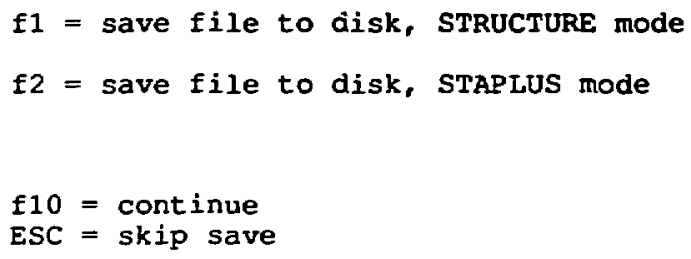

Once you end the data-entry session, you may save the data inso disk files in two different forms.

f1: Saves file to disk, STRUCTURE mode.

2: Saves file to disk, STAPLUS mode.

As you make each selection, the code will ask you to enter a name for the output file. If you do not give an extension to the file name, you will get the default of Jev for the stncure file and stp for the STAPLUS file. The structure file is always written with the energy in keV and either J or $2 \mathrm{~J}$ for the selected spin. The STAPLUS file is always written with the energy in MeV and the spin in J.

When you select to save a file to disk, the following form is displayed:

Enter output file name

Type in the output file name (Enter). The name must have eight or fewer characters. The file extension is optional; however, if none is given the default extensions are lev for the structure file and .stp for the STAPLUS file. If the named file already exist on the disk, it will be overwritten.

Either or both of the output files may be saved. Examples of each are shown in Tables 1 and 3 and in the Appendices. 


\section{Section 4. Branching Fractions Calculations Mode}

\begin{tabular}{|l|}
\hline Select Operating Mode \\
\hline $\mathrm{f} 1=$ Data File Error Analysis \\
$\mathrm{f} 2=$ Interactive Data File Generation \\
$\mathrm{f} 3=$ Eranching Fractions Calculations \\
$\mathrm{f} 4=$ Data File Editing \\
$\mathrm{ESC}=$ Terminate
\end{tabular}

Press $f 3$ for the Branching Fractions Calculations mode. 


\section{Introduction}

This mode of operation, termed the Branching Fractions Calculations mode, is used to compute estimates of multipole transition probability fractions for the depopulation of specific excited nuclear levels, regardless of the extent to which any gamma ray may be internally converted. For each excited level that decays (the ground state and levels specified as "isomeric" are excluded), the branching fractions sum to unity. Thus, when the branching fractions are used in a nuclear reaction code such as STAPLUS, the computed "gamma-ray spectra" may require modification, depending upon the magnitude of the transition energy and the multipole type. Furthermore, only E1, M1, and E2 transitions are calculated here; in most situations this should cause no problems, except possibly if EO transitions are of interest. No particular interactions among multipole types are considered, nor is the decline, with increasing energy, of the accuracy of the K-quantum number restrictions for deformed nuclei.

The general scheme followed in this mode is as follows:

(1) To read in a set of nuclear levels plus their header card, and then

(2) To calculate the relative strengths of up to $\mathbf{3 0}$ of the strongest transitions to lowerenergy levels, conserving angular momentum and parity, for dipole and E2 transitions from radiating levels.

We begin with the highest-energy initial level and then search first for transitions to the ground state and then for transitions to the excited final levels as their excitation energy increases and approaches the energy of the initial state. Up to 20 dipole and 10 E2 transitions are located: if an M1 and an E2 transition populate the same final level, their strengths are added. Finally, should more than $\mathbf{1 0}$ intense transitions be located, their number is truncated to 10 , and the branching fractions are created by normalizing the total strength to unity.

The relative transition strengths are derived from transmission coefficients of the form

$C E 1 \cdot E_{\gamma}^{3}, C M 1 \cdot E_{\gamma}^{3}$ and $C E_{2} \cdot E_{\gamma}^{5}$ where $E_{\gamma}$ is the difference between the initial and final level energies. The coefficients CE1, CM1, and CE2 are constants, except for CE1, which may be either constant or a function of $\mathrm{E}_{\boldsymbol{\gamma}}$. These coefficients are equivalent to gamma-ray strength functions; they may be either relative or absolute in magnitude and may be $\geq 0$. Because the branching fractions are normalized, relative values of the coefficients are most often used. These may either be supplied by the user or calculated from the Weisskopf relative values:

$\begin{array}{lll}C E 1 & =1 . \\ C M 1 & =0.31 \cdot A^{-2 / 3} \\ C E 2 & =\left(7.2 \times 10^{-7}\right) \cdot A^{2 / 3}\end{array}$

Here, $A$ is the nuclear mass number.

In Appendix A, we give our preference for these strength-function values and derive the following approximate expression for the energy-dependent coefficients relative value:

$$
\operatorname{CE} 1\left(E_{\gamma}\right)=k \cdot E_{\gamma} \cdot\left[D_{1}\left(E_{\gamma}\right)+D_{2}\left(E_{\gamma}\right)\right]
$$

The functions $D_{1}$ and $D_{2}$ reffect the properties of the double-(or single-) peak giant dipole resonance (GDR):

$$
D_{i}\left(E_{\gamma}\right)=\frac{\Gamma_{R i}}{\left(E_{\gamma}-E_{R_{i}}\right)^{2}\left(E_{x}+E_{R i}\right)^{2}} .
$$

Here, $\Gamma_{\mathrm{Ri}}$ and $E_{\mathrm{Ri}}$ are, respectively, the width and the energy of the $\mathrm{i}^{\text {th }}$ peak of the GDR, and $\mathrm{E}_{\mathrm{X}}$ is an adjustable constant that we have fixed ${ }^{5}$ at 11 . The units are $\mathrm{MeV}$ for all variables. 
The normalizing constant, $k$, forces $C E 1\left(E_{\gamma}\right)=C E 1$ for $E_{\gamma}$ equal to an input of $E I$ in $M e V$ :

$$
k=\frac{C E 1}{E I} \cdot\left[\frac{1}{D_{1} E_{1}+D_{2}(E !)}\right] \text {. }
$$

Note that the function $D_{i}$ has a singularity at $E_{\gamma}=E_{R i}$; therefore, the highest energy in the discrete level set must be lower than the energy of the first GDR peak. For a single-peak GDR, the values of $\Gamma_{R 2}$ and $E_{R 2}$ should be set to zero.

If the code cannot find any dipole or $\mathrm{E} 2$ transitions that will depopulate a level, it will term that level to be an "isomer." In addition, the user may specify up to six levels, above the ground state, to be isomers, and the code will skip these levels during the multipole decay analysis. The tire frame of the user's problem will dictate which (if any) of these isomers should be retained as such. Any changes in isomer designation or mode of decay must be incorporated by the user. A situation that is not handled by NUSTART occurs when a nuclear level may undergo both radiative and nonradiative decay. For example, if a level undergoes beta decay as well as multipole transitions, the correct multipole branching fraction total is less than unity. The user must account for this in some way, such as by declaring the level to be an isomer and editing the results produced by the reaction code that utilizes the output from NUSTART. The same procedure might be followed with $\rightarrow 0^{+} \rightarrow 0^{+}$transitions, which are not recognized by NUSTART.

Some comparisons of the isomer populations calculated by our nuclear reaction code STAPLUS for reactions on isotopes of bismuth and iridium have been documented 6 using both experimentally based and NUSTART-calculated multipole branches. So far, the identification of potential isomers and their calculated populations have been quite similar using either branching set, for a given discrete level set. How well the populations calculated with either branching set agreed with experiment seemed to depend upon the completeness and accuracy of the discrete level set itself.

In Table 4, we show an example taken from Ref. 6, where two sets of multipole branches among the lower levels of ${ }^{208} \mathrm{Bi}$ are compared. 
Table 4. Comparison of multipole branches among the first 28 out of a total of 87 discrete levels for ${ }^{208} \mathrm{Bi}$. Set 1 is based on experiment ${ }^{7}$ and Set 2 was calculated by NUSTART using the following constant, relative values for the coefficients: $C E 1=1, C M 1=2.7, C E 2=0.03$.

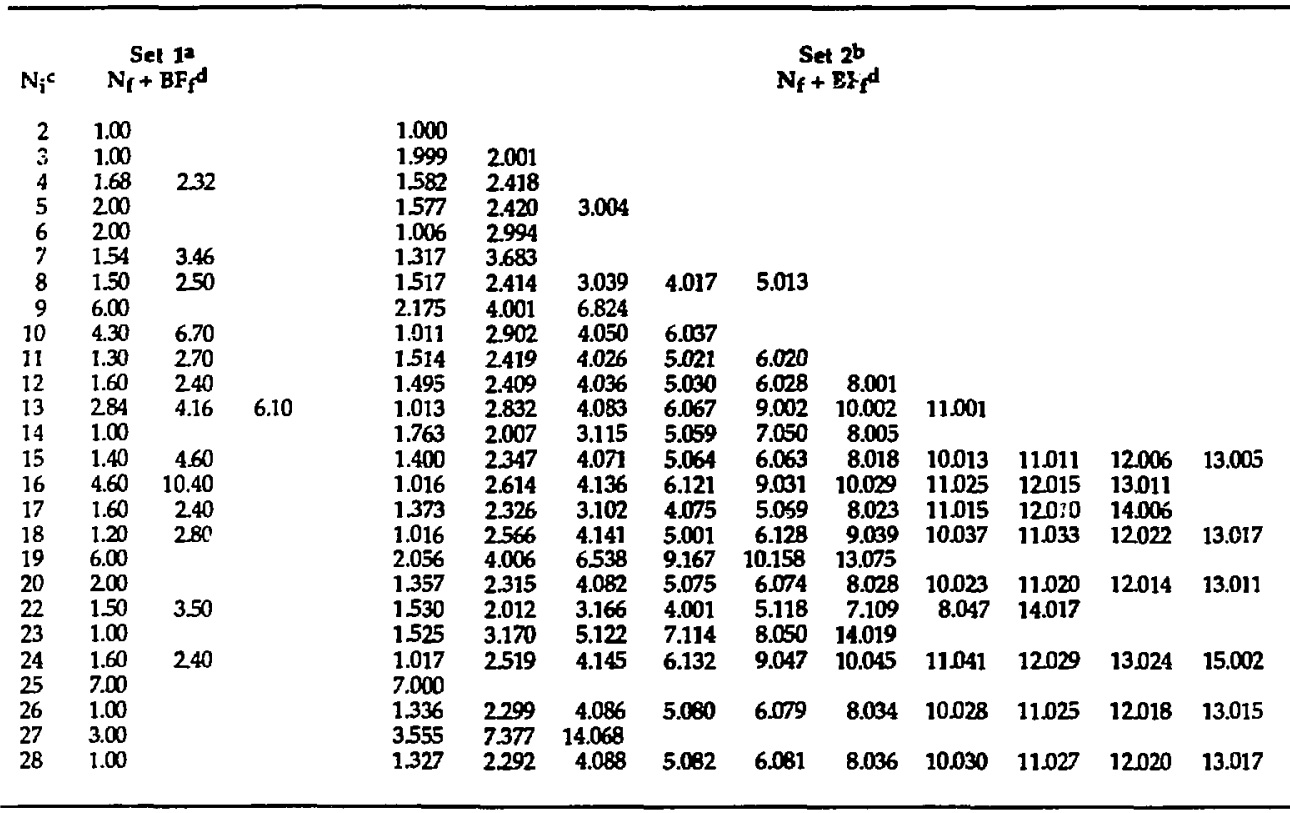

aUsing original branching ratios.

bBranches computed by the LCHECK code.

cNumber of initial radiating level $(1=$ ground state, 2 = first excited state, etc.).

$\mathrm{d}_{\mathrm{f}}$ is the number of the level reached by the transition, and $B F_{f}$ is the fraction of all transitions from level $N_{i}$ that terminate at $N_{f}$. When $B F_{f}=0$, the branching fraction to level $N_{f}$ is unity.

Level 21 corresponds to the $10^{-}$isomer at $1.57 \mathrm{MeV}$ in ${ }^{208} \mathrm{Bi}$, populated to the extent of about $14 \%$ of the total ${ }^{209} \mathrm{Bi}(n, 2 \mathrm{n})$ reaction cross section for an incident neutron energy of about $14 \mathrm{MeV}$. Results using either Set 1 or Set 2 branches agree to within 10\%. Note that the isomer was located and deleted from Set 2 by NUSTART. Although the NUSTART branches yield the proper isomeric population, it is obvious that each parent level has been assigned many more branches than are contained in Set 1 . Thus, the gamma-ray spectrum computed from each set might differ significantly. Comparing calculated with measured gamma-ray spectra, say from an $\left(n, n^{\prime}\right)$ reaction, might provide information about the discrete level set-missing levels, incorrect spin and/or parity assignments, the appropriateness of the nuclear structure theory when the levels are calculated sets, etc. A preliminary study along these lines is mentioned in Ref, 6.

If the nucleus in question is a rigid rotor, the NUSTART code should not be used to calculate multipole transitions among the complete set of levels. The levels that are bandheads should be extracted from the complete level set, and only transitions among these bandheads should be calculated by NUSTART. The user may assume that $\mathrm{K}$ is a good quantum number and require that the K-quantum number restrictions for change be followed: $\Delta K=0, \pm 1$, or $\Delta K=0, \pm 1, \pm 2$ for 
dipole and $\mathrm{E} 2$ transitions, respectively. Up to 50 cases where $K \neq \mathrm{J}$ may be signaled, and the actual $\mathrm{K}$ value for these levels read in. The level numbers in the output file for STAPLUS will not correspond to the true numbers in the complete set from which the bandheads were taken, so provision is made to renumber the levels.

Should the quasi-particle energies in the deformed, Nilsson basis set that create the bandheads be uncertain to a considerable extent, the existence of possible isomeric states and their position with respect to other bandheads may be in doubt. To facilitate sensitivity studies using large sets of calculated levels, we allow a "sensitivity test" option in which all levels of a given parity and with an angular momentum equal to or larger than a specified value of I may be shifted in energy.

One final comment might be made regarding the energy-dependent coefficient option for El transitions. For transition nuclei, those between rigid rotors and closed-shell nuclei, the Kquantum number may rapidly lose its validity, and the onset of band mixing may occur. The large intraband $M 1$ and $E 2$ transition probabilities, that is, the energy-reduced $B(M 1)$ and $B(E 2)$ values, will decrease for the higher-energy levels. It might be possible to simulate this effect by allowing the $\mathrm{E} 1$ transition probabilities to increase with energy faster than those for M1 and E2 transitions, since the sum of the branching fractions is normalized to unity. 


\section{Procedure}

Enter input file name

Type in the input file name (Enter). If the file cannot be found, you will be asked to reenter the file name. Pressing either the ESC key or the f10 key will return you to the calling menu.

$\mathrm{fl}=$ Run Sensitivity Test

f10 = Continue without running Sensitivity Test

ESC = Return to Calling Routine

You can run a sensitivity test on the data by setting the sensitivity parameters. The energy levels that meet the test criteria have an arithmetic operation performed on them. You may either add or subtract some constant from the level energies, or multiply or divide the kevel energies by some constant. The data are then resorred on the level energies.

Press f 1 to bring up the menu for setting the sensitivity parameters.

f1: Runs Sensitivity Test.

Press flo to continue and to calculate the branching fractions without executing the sensitivity test.

f10: Continues without running Sensitivity Test.

ESC: Returns to Calling Routine. This will take you back to the main menu. 
SET SENSITIVITY PARAMETERS

Set Spin Sensitivity greater than 8.0

Set Parity Sensitivity +-

SUBTRACT constant, in MeV, from energy

constant $=0.280$

Press $f 1$ to change setting

Use UP and DOWN ARROW keys to move cursor

$f 10=$ to Continue

ESC $=$ Return to Calling Routine

You can perform the following arithmetic op?rations on selected level energies:

(1) Add a constant to the energy.

(2) Subtract a constant from the energy.

(3) Multiply the energy by a constant.

(4) Divide the energy by a constant.

An energy level to be operated on is marked by comparing its spin and parity against the settings made on this form.

To change the settings, use the UP and DOWN arrow keys to move the cursor to the chosen setting. There are five possible settings:

(1) Set spin sensitivity greater or less than some value N.N. The default setting is greater than N.N. Use the f1 key to toggle between the settings.

(2) Set the spin parameter by typing in a new value (Enter). The default value is 8.0.

(3) Set the parity to compare against. Use the f1 key to step through the possible settings. The parity setting may be negative, positive, or both. The default is both.

(4) Set the chosen arithmetic operation. Use the f1 key to step through the options. Subtraction is the default operation.

(5) Set the constant to use for arithmetic operations. Type in the new value (Enter). The default constant is 0.28 .

When all the settings are correct, press f10 to do the analysis, make any changes, and resort the data. 
For example, if you make the following settings:

Set Spin Sensitivity less than 5

Set Parity Sensitivity -

ADD constant, in $\mathrm{MeV}$, to eirergy

Constant $=0.580$

Then, whenever the spin of an energy level is less than 5 and the parity is negative, the level energy will have 0.58 added to it. After all the energy levels have been analyzed, the data are resorted on the level energies.

Only E1, $M 1$ and E2 transition branches

will be considered

Press flo to continue

Press ESC to go back to previous menu

This is informational only; press $\mathrm{f} 10$ to continue.

Press fl to toggle

Are the Multipole Strength Function

Coefficients (relative or absolute are both OK)

to be supplied by the user? YES

or should the Weisskopf values be used? NO

Press flo to continue

Press ESC to go back to previous menu

The $f 1$ key acts as a switch to toggle between user-supplied coefficients and the Weisskopf values. 


\section{Set Coefficients}

CEI $=0.0000$

$\mathrm{CMI}=0.0000$

$\mathrm{CE} 2=0.0000$

Is CEI energy dependent - YES

Press $f 1$ to toggle setting

Press $\mathrm{f} 10$ to continue

Press ESC to return to previous menu

Type in the coefficient values (Enter).

The highlight bar will automatically move to the next setuing as you enter the values; altematively, use the Arrow keys to move the highlight bar.

To toggle the CE1 energy-dependence seuing, move the highlight bar to that line and press the f1 key.

\begin{tabular}{|l|}
\hline Enter Resonance Parameters \\
\hline EI $=0.0000$ \\
ER1 $=0.0000$ \\
ER2 $=0.0000$ \\
R1 $=0.0000$ \\
R2 $=0.0000$ \\
Press f10 to continue \\
Press ESC to return to previous menu
\end{tabular}

Type in the resonance parameters (Enter).

This form is called up when CE1 is chosen to be energy dependenL. See the Introduction for the parameter definitions. The highlight bar will automatically move to the next seuing as you enter the values; alternatively, use the Arrow keys to move the highlight bar. 
Enter MASS Number 000

Press $f 10$ to continue

Press ESC to go back to previous menu

When the Weisskopf values are used, you must give the mass number for the isolope:

MASS (Enter)

Press fl to toggle

Are the $K$ - Quantum Number Selection Rules

to be considered?

Press flo to continue

Press ESC to go back to previous menu

Use the f 1 key to change the seuing for the selection rules. 
Press fi to toggle

Are the K - Quantum Number Selection Rules

to be considered?

Discrete Level Input will be assumed to be only Rotational Bandheads

Do any Bandheads have $K$ not equal $J$ ? No

Press flo to continue

Press ESC to go back to previous menu

When you chouse to use the K-Quantum number selection niles, you must well if any bandheads have $\mathrm{K}$ not equal to J. If they do, use the fl key to toggle your response. 
When there are some bandheads with $\mathrm{K}$ not equal to J, the following form will appear:

Enter Level Numbers and Positive ' $K$ ' Values

(as blank delimited pairs)

A maximum of 50 levels may be entered

Press $\mathbf{f 1 0}$ to continue Press ESC to cancel and return

Type in the level numbers and the $K$ values as blank delimiled pairs in the form:

level $\mathrm{K}$ level $\mathrm{K}$ level $\mathrm{K} \ldots$

The "level" is the level number ( 1 = ground state, etc).

The $\mathrm{K}$ value is to be given as a decimal number.

Press f10 to end the data entry and go on to the next form.

Press ESC to nullify any data entered and return to the calling form. 
Press fl to toggle

Are the BANDHEADS to be renumbered? No

Press $\$ 10$ to continue

Press ESC to go back to previous menu

If the II key is used to change NO to YES, a subsequent menu will ast for the information required to accomplish the renunbering.

Press fl to toggle

Are there any IsOMERS present in the data? No

Press flo to continue

Press ESC to go back to previous menu

Use the fl key to toggle your response. 
Enter Level Numbers for Isomers (blank delimited)

(a maximum of six levels)

Press flo to continue Press ESC to cancel and return

When there are isomers present, you can enter the isomer level numbers. Up to six isomer levels may be entered. Press either the Enter key or the f 10 key to terminate input.

Due to the approximate nature of the physics

involved, no more than 10 branches will be

calculated for each level

Press $f 10$ to continue

Press ESC to go back to previous menu

This is informational only; press f10 to continue and to calculate the branching ratios. 
Select Input Mode for Renumbering Levels

$f l=$ Read New Level Numbers from a Disk File

f2 = Read New Level Numbers from Terminal Input

f3 = Prompt for each New Level Number

ESC $=$ Cancel Renumber and Return to Main Menu

When tive option to renumber the bandheads is chosen, the above menu will be displayed. You have three options for passing the renumbering information. These options are described below. Either press the proper function key to select the input mode wanted or press the ESC key to cancel the renumbering of the bandheads, to complete the branching fractions calculations, and to reum to the main menu.

Enter input file name

If a disk file is to be used for entering the level values for renumbering, type in the input file name (Enter).

The input file cortains a list of the bandhead option (old) level numbers and the true (new) level numbers as blank delimited data pairs. For example.

$\begin{array}{llllll}\begin{array}{c}\text { old } \\ \text { level }\end{array} & \begin{array}{l}\text { new } \\ \text { level }\end{array} & \begin{array}{c}\text { old } \\ \text { level }\end{array} & \begin{array}{c}\text { new } \\ \text { level }\end{array} & \begin{array}{c}\text { old } \\ \text { level }\end{array} & \begin{array}{c}\text { new } \\ \text { level }\end{array} \\ 1 & 1 & 2 & 2 & 3 & 4 \\ 4 & 10 & 5 & 11 & 6 & 13 \\ 7 & 15 & \text { eic. } & & & \end{array}$

There are no constraints regarding column placement or the number of pairs on a line. The file must contain NDISK pairs of numbers. Even if the level number is not to be changed, a pair of numbers (the old number repeated) must appear for every level from 1 to NDISK. 


\section{Enter NDISK Old and New Level Numbers as Blank Delimited Pairs}

old new old new old new old new old new level level level level level level level level level level

Jse ARROW KEYS to move Cursor

$\mathrm{f} 10=$ to continue

ESC = Cancel Data Entry and Return to Main Menu

Enter the bandhead option (old) and the true (new) level numbers through the terminal. Use either the Enter key or the space bar to terminate each number as it is entered. As each number is entered, the cursor will automatically move to accept the next number.

The UP, DOWN, LFFT, and RIGHT arrow keys allow you to move back through the energy numbers for modification. To change a level number, move the cursor to the number to be changed, type in the new number and (Enter) or (Space).

Press fio to terminate the data entry. NUSTART will then change the level numbers to the new values and will write the revised output to the end of the STT file.

Press the ESC key to cancel the call to renumber the bandheads and to continue with the normal execution of the program.

Remember-there must be NDISK pairs of numbers. 


\begin{tabular}{l} 
Enter New Level Number After Prompt \\
old new old new old new old new old new \\
level level level level level level level level level level \\
\hline 1 \\
Use ARRDW KEYS to move Cursor \\
ESC $=$ to continue Cancel Data Entry and Return to Main Menu
\end{tabular}

With this option, the bandhead option (old) level numbers are printed out as a prompt, and you must enter the true (new) level number (Enter). Use the UT, DOWN, LEFT, and RIGHT arrow keys to move around through the new level numbers for editing. To correct the energy number, move the cursor to the value to be changed and type in the new value (Enter). file.

Press f10 to terminate the data entry. The revised data are written to the end of the stp

Press the ESC key to cancel the bandhead renumbering and to continue with the normal execution of the program. 


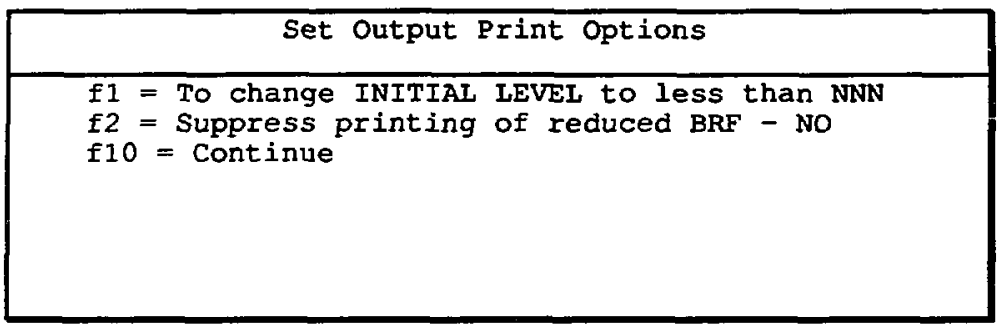

Once the branching fractions have been calculated, NUSTART runs an analysis on the results and writes the calculated branching fractions and the data-analysis table to the dataanalysis file.

Once the analysis is complete, you can set the print options for the output.

Press $\mathrm{fl}$ to type in the number of branching levels that will be printed. The default is all of the levels; this is shown when the form comes up (NDISK). If you decide you do not want the data-analysis table written, set the INITIAL LEVEL to 0.

f1: Changes INITIAL LEVEL to less than NNN.

$$
\text { * * NOTE * * * }
$$

NNN is replaced by the actual number of levels in the file when the form is displayed.

Use the toggle switch $\mathrm{f} 2$ to control the printing of the BRF information. The default is to print everything.

22: Suppresses printing of reduced BRF - NO.

Continue on with the next step. The data-analysis file will be written and you will be returned to the main menu for the next option.

f10. Continues.

These reduction options are particularly useful if the data-analysis file is too large to be written onto a floppy disk. 


\section{Section 5. Data File Editing}

\begin{tabular}{|l|}
\hline \multicolumn{1}{|c|}{ Select Operating Mode } \\
\hline$f 1=$ Data File Error Analysis \\
$\mathrm{f} 2=$ Interactive Data File Generation \\
$\mathrm{f} 3=$ Branching Fractions Calculations \\
$\mathrm{f} 4=$ Data File Editing \\
$\mathrm{ESC}=$ Terminate
\end{tabular}

Press $\mathrm{f} 4$ to edil any standard STAFíUS data file. 


\section{Introduction}

You may edit the header card and one or more levels of an input file and change any of the data parameters for that level, including the branching fraction information. No levels may be added or deleted. You may open an input file that contains only the discrete levels and no multipole branches (for the Branching Fractions Calculations mode), a file with the discrete levels plus multipole branches (for the Data File Error Analyses mode), or the STAPLUS output file from the bandhead option of the third mode with the appended set of renumbered levels for the multipole branches.

First, you must tell NUSTART which file you are going to edit. You then select the level you want to edit. You may then edit any or all of the data parameters for that level. You can step up and down through all the levels in the file, editing any that you want. However, because the editor was not designed to be sophisticated, you are limited to moving through the file one level at a time. Therefore, if you need to edit two widely separated levels, you may want to edit one level, end the editor, and restart it to edit the second level.

Besides allowing you to edit files, the edit mode can be used to create a structure-formatted file from any standard STAPLUS input file. Open any STAPLUS file to any arbitrary level for editing and immediately end the editing mode. When you end the edit mode, you are given the option of writing a structure-format file.

You can also use the edit mode to split up batch files into their component parts by writing each component to a separate file.

In all cases, the editor does not modify your original input file. All modifications are done in memory; the results are writter: to a new disk file, which you name. 


\section{Procedure}

$$
\begin{aligned}
& \mathrm{fl}=\text { open new file to edit } \\
& \mathrm{f2}=\text { edit next file in batch file } \\
& \mathrm{ESC}=\text { return to main menu }
\end{aligned}
$$

Once you have selected the edit option, you must point to where the file is.

f1: Opens a new disk file and allows you to edit it.

f2: Allows you to edit the next data set in the file when you are working with a batch file.

When a new file is to be opened, the following form is displayed:

$$
\text { Enter input file name }
$$

Type in the input file name (Enter). 
Enter nucleus

Press flo to continue

Press ESC to go back to previous menu

Type in the name of the nucleus (Enter).

Enter: Acts as a terminator for the input and steps to the next form.

Press $\mathrm{f} 10$ to continue.

f10: Steps to the next form without changing any current settings.

Press ESC to go back to the previous menu.

\section{Enter comment}

Press $f 10$ to continue

Press ESC to go back to previous menu

Type in any comment you want put in the output file on card 1 . The comment may have a maximum of 45 characters.

Press the Enter key to terminate the comment entry and to step to the next form.

Press fio to continue.

f10: Steps to the next form without causing any change in any current setting.

Press ESC to go back to the previous menu. 
There are NDISK levels

Enter level number to edit

Press $f 10$ to continue

Press ESC to go back to previous menu

Type in the level number to edit (Enter). You may start at any level; use the $f 9$ and $f 10$ keys to step up or down through the levels (see below for more detail). 


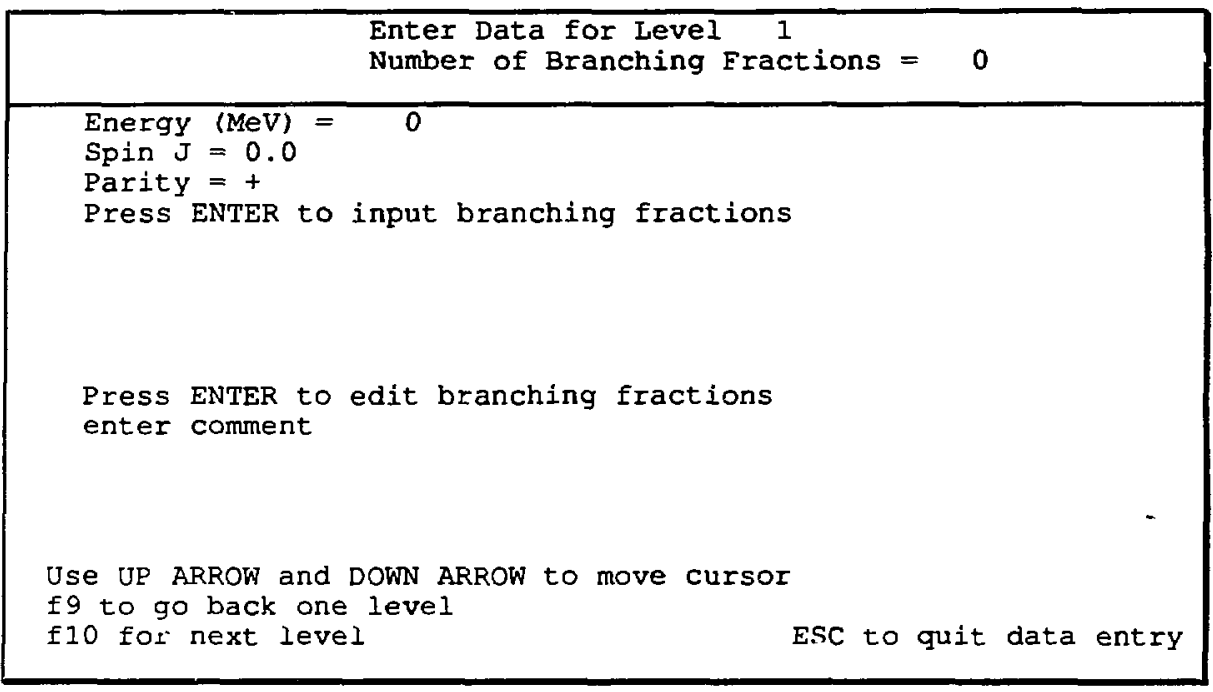

This is the main form for editing the data files. The first line shows the current level number. The second line shows the current number of branching percentages for the level.

As you fill in the form, the active option is shown by a highlight bar. When you fill in a option, the highlight moves down to the next entry. You can also move up and down through the form by using the Up and Down arrows to move the highlight bar.

Energy $(\mathrm{MeV})=0$

To enter the energy, type in the value (Enter) in MeV.

Spir $\mathrm{J}=0.0$

To set the spin, type in the spin value (Enter).

Parity $=+$

The parity is set by using the f1 key as a toggle switch.

Press Enter to input branching fractions (as percentages).

To input the branching percentages, press Enter. A new form will pop up to allow you to type in the branching percentage values (see below). 
Press Enter to edit branching fractions.

If any of the branches are incorrect, you can edit them by bringing up the editing form. The editing form shows the bianches in columns of energy level and percent of decay. Ensure that the branching percentages sum to 100 .

Use the arrow keys to move the cursor to the value to be changed. You may move through the branching fractions at will, and change them in any order.

Press $\mathrm{fl}$ to open the value for change.

Type in the new value (Enter).

Press f10 to end the editing and to return to the data entry form.

You may cancel the editing session at any time and nullify any changes made by pressing the ESC key.

\section{Enter comment.}

To put a comment in the output file for the current level, type in the comment (Enter). This comment will be written after the branching fractions. The comment may have a maximum of 45 characters.

Press $f 9$ to go back one level.

49: Moves you down to the previous level. You may change any value in this level.

Press $\mathrm{f10}$ for the next level.

f10: Moves you to the next highest level.

When you have filled in the data for all the levels wanted, press the ESC key to quit.

ESC: Quits data entry. 
When you select to input branching percentages, the following form is brought up:

Enter Branching Percentages

(as blank delimited pairs)

a maximum of 30 branching percentages may be entered

Press f10 to continue

Press ESC to cancel and return

To enter the branching percentages, type them in as blank delimited pairs in the form:

level_energy \%_decay level_energy \%_decay ...

The level_energy is entered in $\mathrm{MeV}$. The percent decay may be entered as whole numbers or as decimal values (e.g., 33, 33.3, .03, etc.). When all the branching ratios are in, press f10 to quit and retum. You may, at any time, nullify the data entry and return to the calling form by pressing the ESC key.

\section{- NOTE}

The maximum value for the energy is $9.999 \mathrm{MeV}$. The percent of decay can have a maximum of four characters (e.g., 33.3, 22.5, etc.) any value greater than 100 percent will be treated as 100 . 
When you select to edit the branching percentages, the following form is brought up:

Branching percentages for level 14

EI EI $\quad$ EI

Use the cursor arrows to move cursor to value to be changed I1 to change value

f10 to return

To edit the branching ratios:

(1) Use the arrow keys to highlight the value to be changed.

(2) Press the f1 key to toggle the program to accept the change.

(3) Type in the new value (Enter).

Repeat these steps for each change to be made. When you have made all your changes, press the 110 key to continue.

Press the ESC key to nullify all changes and to cancel the editing option. 


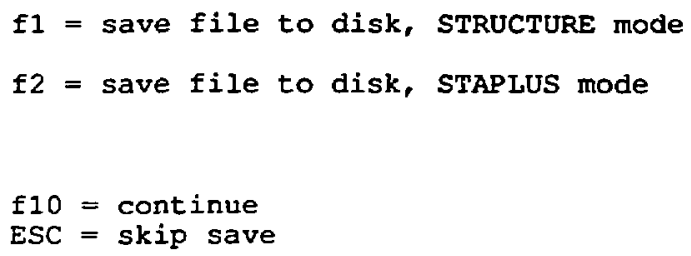
forms.

Once you end the data-entry session, you may save the data into disk files in two different

f1: Saves file to disk, STRUCTURE mode.

2: Saves file to disk, STAPLUS mode.

As you make each selection, the code will ask you to enter a name for the output file. If you do not give an extension to the file name, you will get the default of lev for the structure file and .stp for the STAPLUS file. The structure file is always written with the energy in keV. The STAPLUS file is always written with the energy in MeV. The sin is in J.

Either or both of the output files may be saved; examples of each have been shown in Tables 1 and 3.

Enter output file name

Type in the output file name (Enter).

The output file name may have a maximum of eight characters plus the extension. As you make each selection, the code will ask you to enter a name for the output file. If you do not give an extension to the file name, you will get the default of lev for the structure file and stp for the STAPLUS file. If the file already exists on the disk, it will be overwritten. 


\section{References}

1. M. Uht and B. Strohmaier, A Computer Code for Particles Induced Actioation Cross Sections and Related Quantities, Institute für Radiumforschung und Kemphysik Report IRK 76/01 with Addenda (1976).

2. G. L. Larsen, M. A. Gardner, and D. G. Gardner, Development of IBM PC Codes for Use in Our Nuclear Cross-Section Caiculational Effort, Lawrence Livermore National Laboratory Report UCAR-10062-88 (1988), p. 50.

3. R. W. Hoff et al., Nucl. Phys. A437, 285 (1985).

4. S. Brant, V. Paar, and D. Vretenar, Z. Phys. A319, 355 (1984).

5. D. G. Gardner, M. A. Gardner, and R. W. Hoff, "Absolute Dipole Gamma-Ray Strength Functions for ${ }^{176} \mathrm{Lu}$," in Proc. 5th Int. Symp. Capture Gamma-Ray Spectroscopy and Related Topics, Knoxville, TN, September 1984, p. 513; also Lawrence Livermore National Laboratory Report UCRL-91098, and Supplement (1984).

6. D. G. Gardner, M. A. Gardner, and G. L. Larsen, Completion and Applicotions of the PC Code LCHECK, Lawrence Livermore National Laboratory Report UCAR-10062-89, p. 43.

7. R. A. Meyer, Lawrence Livermore National Laboratory, private communication (1985).

8. D. G. Gardner, "Methods for Calculating Neutron Capture Cross Sections," in Neutron Radiative Capture (R. E. Chrien, Ed.), Vol. 3, Chapter III (Pergammon Press, Oxford, 1984).

9. D. G. Gardner, M. A. Gardner, and F. S. Dietrich, A Study of Gamma-Ray Strength Functions, Lawrence Livermore National Laboratory Report UCID-18759 (1980). 


\section{Appendix A. Energy-Dependent E1 Multipole Strength Function}

By invoking the Brink-Axel hypothesis, the classical dipole sum rule, and a functional form, $G_{R}$, for the giant dipole resonance (GDR), the E1 strength function may be written as

$$
f_{E 1}\left(E_{\gamma}\right)=3.32 \times 10^{-6}\left(\frac{N Z}{A}\right) \frac{F_{S R}}{E_{\gamma}} \sum_{i} \frac{G_{R i}}{\Gamma_{R i}} \mathrm{MeV}^{-3}
$$

Definitions of these terms may be found in Ref. 8; for present purposes, we use the notation in Section 4 of this report, where we defined a relative quantity

$$
\operatorname{CEI}\left(E_{\gamma}\right)=k \cdot \frac{1}{E_{\gamma}} \cdot \sum_{i} \frac{G_{R i}}{\Gamma_{R i}}
$$

Here, $k$ is a normalizing constant, $E_{\gamma}$ is the energy of the dipole transition, and $G_{R}$ is the unit line shape chosen for the GDR. The GDR may have one or two peaks $\left(i=1\right.$ or 2 ), and $\Gamma_{R i}$ is the width of the $i^{\text {th }}$ peak. For the GDR line shape, we choose the energy-dependent Breit-Wigner form 8,9

$$
G_{R}=\left[1+4\left(E_{\gamma}-E_{R}\right)^{2} / \Gamma_{R} \Gamma\left(E_{\gamma}\right)\right]^{-1}
$$

The two new terms are $E_{R}$, the GDR peak energy, and $J\left(E_{\gamma}\right)$, an nnergy-dependent damping width. For $E_{\gamma}<E_{R}$, we have

$$
\Gamma\left(E_{\gamma}\right)=\Gamma_{R}\left[2 E_{\gamma}\left(E_{x}+E_{R}\right)\right]^{2}
$$

As noted earlier, $E_{x}$ is a free variable that we have fixed at $11 \mathrm{MeV}{ }^{5}$ By limiting $E_{\gamma}$ to low energies, the first term, 1, in the square brackets of Eq. (A-3) may be disregarded, leading to the previous Eqs. (4-1) and (4-2).

When NUSTART is used to calculate the branching fractions among the discrete levels, values must be assigned to the constants, CE1, CM1, and CE2 I which represent the (usually relative) multipole strength functions] before they are multiplied by the transition energy raised to the appropriate power. If the E1 transitions are based on some representation of the GDR, the additional information previously cited in Section 4 must be supplied. Because we are only beginning to investigate how nuclei in different mass regions and with different deformations should be treated, the user should consider the following merely as a very rough guide.

We rarely use the Weisskopf estimates, preferring to base our choice of strength-function values on those we calculate for the continuum in a given nucleus, modified in some ad hoc manner for the collective character in deformed nuclei. Usually, we assume that $C E 1=1$, at an excitation energy midway among the discrete levels. If CE1 is based on the GDR description, additional resonance information must be supplied. Then, we check the literature for existing experimental gamma-ray information for nuclei in the mass region of interest. For mediumweight to heavy nuclei, we often find relative strength-function constants that span a considerable range of values. For example, using $C E 1=1$, and about 0.5 to $1 \mathrm{MeV}$ of excitation energy, we obtain the values listed in Table A-1. 
Table A-1. Typical relative strength-function constants used if $\mathrm{CE}=1$.

\begin{tabular}{lcc}
\hline Nuclear type & CM1 & CE2 \\
\hline Deformed & 3 to 6 & 1 to 0.01 \\
Intermediate & 1 to 5 & 0.1 to 0.01 \\
Closed shell & 0.5 to 5 & -0.01 \\
\hline
\end{tabular}

For deformed nuclei, the values in Table A-1 refer to interband transitions; NUSTART should not be used with a complete set of levels from a permanent rotor. 


\section{Appendix B. Sample NUSTART Input and Output Files}

\section{Example 1}

In this example, the input file consisted of the discrete levels of ${ }^{208} \mathrm{Bi}$ and the experimental branching fractions of Set 1 in Table 4. When this file was processed in NUSTA.RT's Data File Error Analysis mode, Table B-1 was generated.

Table B-I. Portion of the data-analysis output file for ${ }^{208} \mathrm{Bi}$.

GAMMA-RAY TRANSITIONS AMONG DISCRETE LEVELS

Initial Level

\begin{tabular}{lllllllllll} 
& & \multicolumn{9}{c}{ Final Level } \\
& E & J & P & & & N & P & EG & BR F
\end{tabular}

\begin{tabular}{|c|c|c|c|c|c|c|c|c|c|}
\hline \multirow{3}{*}{$\begin{array}{r}28 \\
+\quad\end{array}$} & 1.699 & 4.0 & -1 & \multirow{3}{*}{1} & \multirow[b]{2}{*}{0.000} & \multirow[b]{2}{*}{5.0} & \multirow[b]{2}{*}{1} & \multirow[b]{2}{*}{1.699} & \multirow[b]{2}{*}{1.000} \\
\hline & & & & & & & & & \\
\hline & Passible & Multi & poles & & & EI & & M2 & E3 \\
\hline 27 & 1.673 & 7.0 & -1 & & & & & & \\
\hline \multirow{2}{*}{+2} & & & & 3 & 0.510 & 6.0 & 1 & 1.163 & 1.000 \\
\hline & Possible & \multicolumn{2}{|c|}{ e Multipoles } & & & EI & & M2 & E3 \\
\hline 26 & 1.657 & 4.0 & 1 & & & & & & \\
\hline \multirow[t]{2}{*}{+} & & & & 1 & 0.000 & 5.0 & 1 & 1.667 & 1.000 \\
\hline & Possible & \multicolumn{2}{|c|}{ Multipoles } & & & M1 & & E2 & M3 \\
\hline & 1.664 & 8.0 & -1 & & & & & & \\
\hline \multirow{2}{*}{$\begin{array}{r}25 \\
+\quad\end{array}$} & & & & 7 & 0.651 & 7.0 & 1 & 1.013 & 1.000 \\
\hline & Possible & \multicolumn{2}{|c|}{ Multipoles } & & & E1 & & M2 & E3 \\
\hline 24 & 1.637 & 3.0 & 1 & & & & & & \\
\hline \multirow[t]{3}{*}{+} & & & & 2 & 0.063 & 4.0 & 1 & 1.574 & 0.400 \\
\hline & Possible & Multi & poles & & & M1 & & E2 & M3 \\
\hline & $\mathrm{BR} E / E G^{\star} *$ & * $(2 L+1$ & & \multirow[b]{2}{*}{1.637} & \multicolumn{2}{|c|}{$1.03 e-001$} & \multicolumn{2}{|c|}{$4.14 e-002$} & $1.67 e-002+$ \\
\hline \multirow[t]{3}{*}{1} & 0.000 & 5.0 & 11 & & 0.600 & & & & \\
\hline & Possible & Multi & poles & & & E2 & & M3 & \\
\hline & $B R E / E G^{*} *$ & * $(2 \mathrm{~L}+1$ & & & & $e-002$ & 1.5 & $10 e-002$ & \\
\hline 23 & 1.629 & 6.0 & -1 & & & & & & \\
\hline+ & & & & 1 & 0.000 & 5.0 & 1 & 1.629 & 1.000 \\
\hline & Possible & Multi & poles & & & E1 & & M2 & E3 \\
\hline
\end{tabular}


Initial Level

\begin{tabular}{llllllllll} 
& & \multicolumn{9}{c}{ Einal Level } \\
& E & J & P & & & & & & \\
& & & N & E & J & P & EG & BR F
\end{tabular}

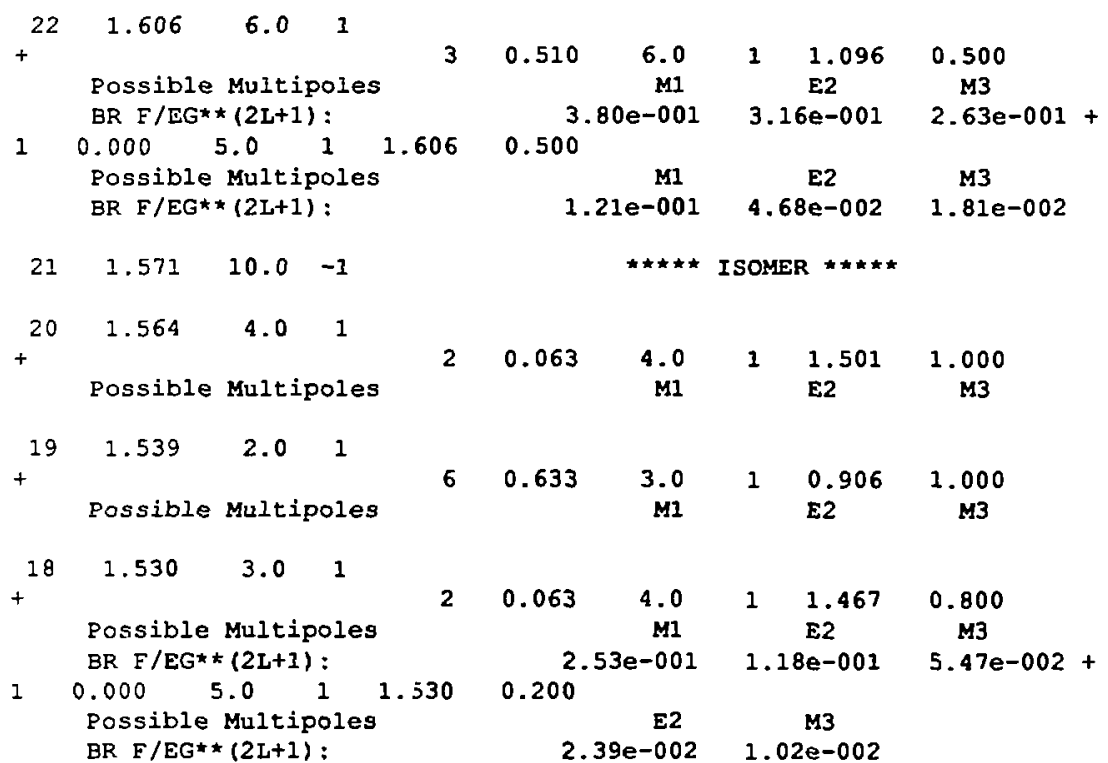

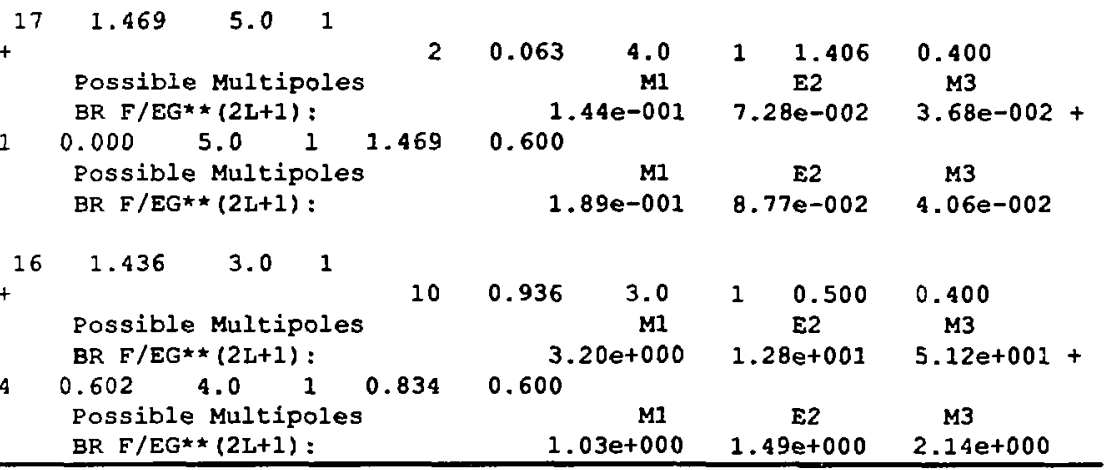


Initial Level

\begin{tabular}{lllllllllll} 
& & \multicolumn{9}{c}{ Final Level } \\
& E & J & P & & & & & & \\
& & & N & E & J & P & EG & BR F
\end{tabular}

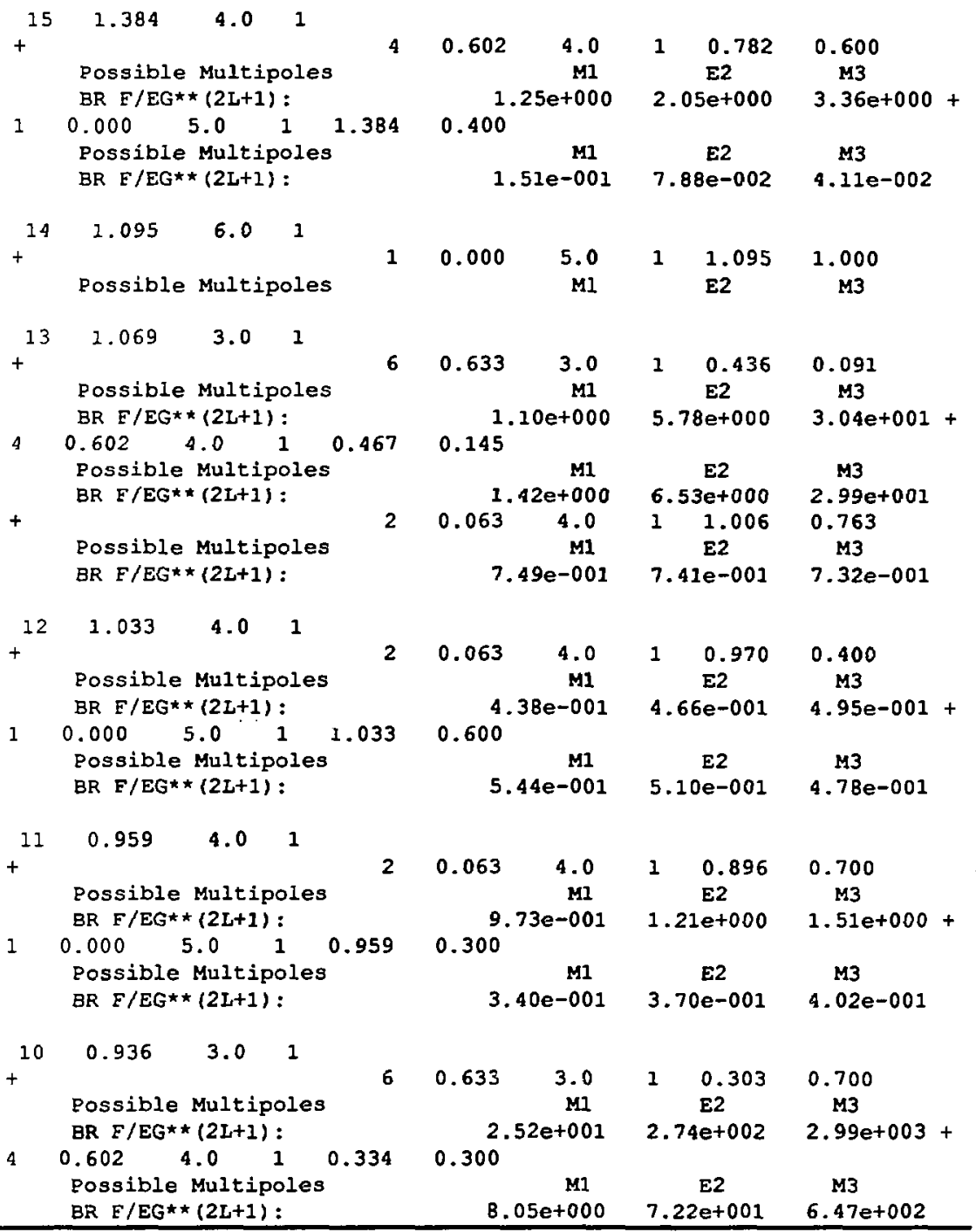


Initial Level

$\begin{array}{llllllllll}\text { N } & \text { E } & \text { J } & \text { P } & & & & & & \\ & & & & \text { N } & \text { E } & \text { J } & \text { P } & \text { EG } & \text { BR F }\end{array}$

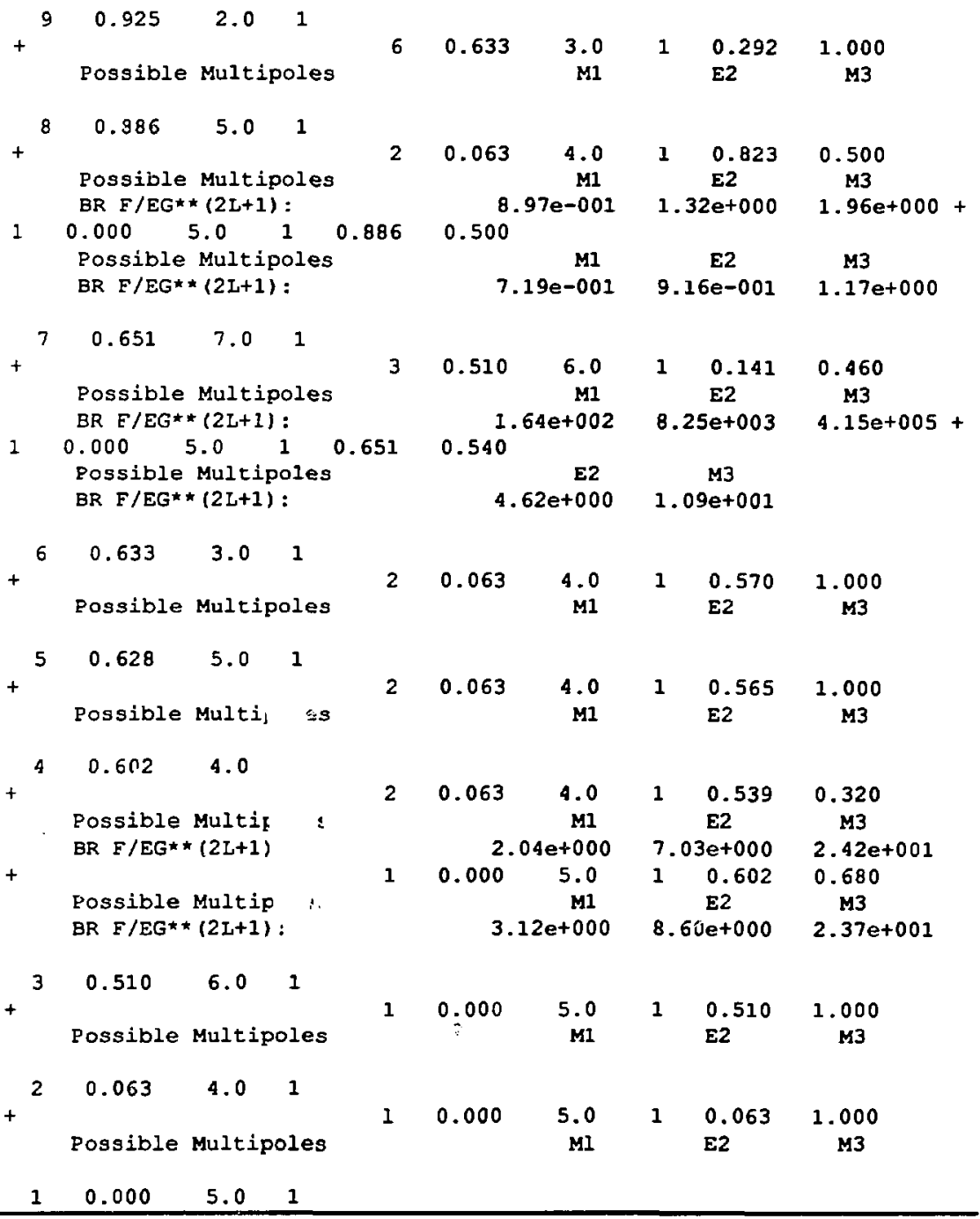




\section{Example 2}

In this example, we took 62 bandheads from a set of 291 discrete levels for ${ }^{176} \mathrm{Lu}$, up to an excitation energy of about $1.5 \mathrm{MeV}$, among which the multipole branches had been calculated 5 using only dipole transitions with CM1/CE1 $=6$. Then, we used the NUSTART code to recalrulate the multipole branches among the band heads. We allowed CE1 to be energy dependent and introduced a significant number of E2 transitions. No levels were designated to be isomers before the start of the new calculation, and NUSTART located level 2 as the only isomer. Finally, the band-head level numbers were changed to their true values in the 291level set via an input file that listed the pairs of bandhead option (old) and true (new) level numbers. In Tables B-2a through B-2h are shown the pertinent input and output information for this NUSTART calculation.

Table B2a. Set of bandhead levels far ${ }^{176} \mathrm{Lu}$.

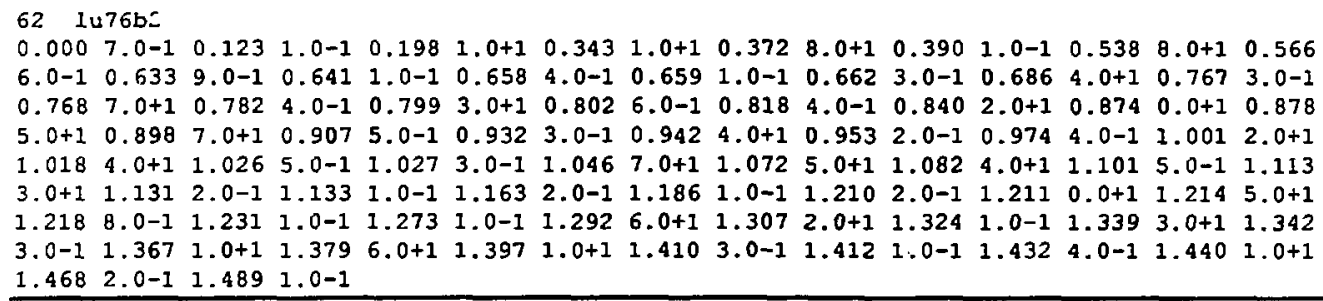

Table B2b. The strength-function parameters that were used for this sample calculation.

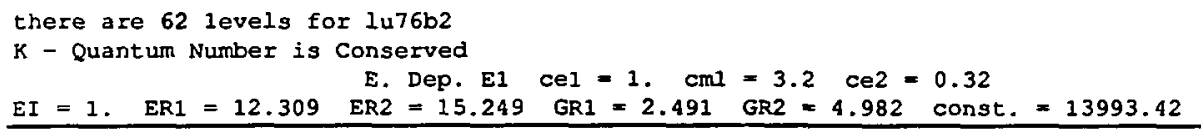

The value of the normalizing constant, $k$, for the energy-dependent E1 transitions was determined by NUSTART to be $13,993.42$. 
Bandhead levels have been set:

level $=2, K=0.0$

level $=12, K=0.0$

level $=40, K=0.0$

level $=42, K=0.0$

level $=62, K=0.0$

ENERGY SPIN PARITY K

(MeV) (J)

$0.000 \quad 7.0 \quad-1$

$0.123 \quad 1.0 \quad-1$

$0.198 \quad 1.0+1$

$0.343 \quad 1.0+1$

$0.372 \quad 8.0+1$

$0.390 \quad 1.0 \quad-1$

$\begin{array}{lll}0.538 & 8.0+1\end{array}$

$0.566 \quad 6.0 \quad-1$

$0.633 \quad 9.0 \quad-1$

$0.641 \quad 1.0 \quad-1$

$0.658 \quad 4.0 \quad-1$

$0.659 \quad 1.0 \quad-1$

$0.662 \quad 3.0 \quad-1$

$\begin{array}{lll}0.686 & 4.0+1\end{array}$

$0.767 \quad 3.0 \quad-1$

$\begin{array}{lll}0.768 & 7.0+1\end{array}$

$0.782 \quad 4.0 \quad-1$

$0.799 \quad 3.0+1$

$0.802 \quad 6.0 \quad-1$

$0.818 \quad 4.0 \quad-1$

$0.840 \quad 2.0+1$

$0.874 \quad 0.0+1$

$\begin{array}{lll}0.878 & 5.0+1\end{array}$

$\begin{array}{lll}0.898 & 7.0+1\end{array}$

$0.907 \quad 5.0 \quad-1$

$0.932 \quad 3.0 \quad-1$

$0.942 \quad 4.0 \quad+1$

$0.953 \quad 2.0 \quad-1$

$0.974 \quad 4.0 \quad-1$

$1.001 \quad 2.0 \quad+1$

$1.018 \quad 4.0+1$

$1.026 \quad 5.0 \quad-1$

$1.027 \quad 3.0 \quad-1$

$1.046 \quad 7.0+1$

$1.072 \quad 5.0+1$

$1.082 \quad 4.0+1$

$1.101 \quad 5.0 \quad-1$

$1.113 \quad 3.0+1$

$\begin{array}{lll}1.131 & 2.0 & -1\end{array}$ 
Table B-2c. (Continued).

\begin{tabular}{llll}
$\begin{array}{l}\text { ENERGY } \\
\text { (MeV) }\end{array}$ & $\begin{array}{l}\text { SPIN } \\
\text { (J) }\end{array}$ & PARITY & K \\
1.133 & 1.0 & -1 & 0 \\
1.163 & 2.0 & -1 & \\
1.186 & 1.0 & -1 & 0 \\
1.210 & 2.0 & -1 & \\
1.211 & 0.0 & +1 & \\
1.314 & 5.0 & +1 & \\
1.218 & 8.0 & -1 & \\
1.231 & 1.0 & -1 & \\
1.273 & 1.0 & -1 & \\
1.292 & 6.0 & +1 & \\
1.307 & 2.0 & +1 & \\
1.324 & 1.0 & -1 & \\
1.339 & 3.0 & +1 & \\
1.342 & 3.0 & -1 & \\
1.367 & 1.0 & +1 & \\
1.379 & 6.0 & +1 & \\
1.397 & 1.0 & +1 & \\
1.410 & 3.0 & -1 & \\
1.412 & 1.0 & -1 & \\
1.432 & 4.0 & -1 & \\
1.440 & 1.0 & +1 & \\
1.468 & 2.0 & -1 & \\
1.489 & 1.0 & -1 & \\
\hline
\end{tabular}


LEVEL NBF

\begin{tabular}{|c|c|c|c|c|c|c|c|c|c|c|c|}
\hline 3 & 1 & 2.000 & & & & & & & & & \\
\hline 4 & 2 & 2.175 & 3.825 & & & & & & & & \\
\hline 5 & 1 & 1.000 & & & & & & & & & \\
\hline 6 & 2 & 2.981 & 3.019 & & & & & & & & \\
\hline 7 & 2 & 1.842 & 5.158 & & & & & & & & \\
\hline$B$ & 1 & 1.000 & & & & & & & & & \\
\hline 9 & 3 & 1.885 & 5.113 & 7.002 & & & & & & & \\
\hline 10 & 4 & 2.830 & 3.064 & 4.013 & 6.093 & & & & & & \\
\hline 11 & 1 & 8.000 & & & & & & & & & \\
\hline 12 & 4 & 2.817 & 3.067 & 4.014 & 6.101 & & & & & & \\
\hline 13 & 1 & 6.000 & & & & & & & & & \\
\hline 14 & 2 & 11.650 & 13.350 & & & & & & & & \\
\hline 15 & 4 & 6.236 & 11.401 & 13.359 & 14.004 & & & & & & \\
\hline 16 & 4 & 1.580 & 5.349 & 7.068 & 8.003 & & & & & & \\
\hline 17 & 4 & 8.013 & 11.514 & 13.466 & 14.006 & & & & & & \\
\hline 18 & 5 & 3.684 & 4.172 & 11.009 & 13.008 & 14.126 & & & & & \\
\hline 19 & 2 & 1.976 & 8.024 & & & & & & & & \\
\hline 20 & 6 & 8.012 & 11.496 & 13.460 & 14.010 & 15.016 & 17.006 & & & & \\
\hline 21 & 4 & 3.666 & 4.304 & 6.028 & 10.001 & & & & & & \\
\hline 22 & 6 & 2.167 & 3.541 & 4.262 & 6.028 & 10.001 & 12.001 & & & & \\
\hline 23 & 4 & B. 255 & 11.062 & 14.680 & 17.002 & & & & & & \\
\hline 24 & 5 & 1.497 & 5.372 & 7.117 & 8.009 & 16.005 & & & & & \\
\hline 25 & 7 & 1.505 & 8.330 & 11.128 & 14.005 & 17.016 & 19.010 & 20.006 & & & \\
\hline 26 & 9 & 6.084 & 10.004 & 11.371 & 13.354 & 14.018 & 15.081 & 17.060 & 18.002 & 20.027 & \\
\hline 27 & $B$ & 11.075 & 13.071 & 14.700 & 15.011 & 17.007 & 18.121 & 20.003 & 23.011 & & \\
\hline 28 & 7 & 2.093 & 3.230 & 4.096 & 6.434 & 10.072 & 13.059 & 15.015 & & & \\
\hline 29 & 9 & 8.013 & 11.370 & 13.356 & 14.022 & 15.104 & 17.083 & 18.003 & 20.044 & 25.003 & \\
\hline 30 & 7 & 3.605 & 4. 326 & 6.045 & 10.005 & 13.004 & 18.009 & 21.005 & & & \\
\hline 31 & 9 & 11.076 & 13.073 & 14.592 & 15.018 & 17.019 & 18.169 & 20.007 & 23.044 & 27.007 & \\
\hline 32 & 9 & 1.373 & 8.326 & 11.166 & 13.002 & 14.012 & 17.048 & 19.037 & 20.030 & 25.006 & \\
\hline 33 & 10 & 6.066 & 10.005 & 11.321 & 13.311 & 14.024 & 15.112 & 17.093 & 18.005 & 20.058 & 26.005 \\
\hline 34 & 7 & 1.432 & 5.367 & 7.154 & 8.018 & 16.025 & 19.001 & 24.004 & & & \\
\hline 35 & 10 & 8.189 & 11.084 & 14.582 & 16.003 & 17.020 & 19.015 & 20.012 & 23.073 & 25.002 & 27.022 \\
\hline 36 & 10 & 11.076 & 13.073 & 14.517 & 15.023 & 17.019 & 18.187 & 20.011 & 23.070 & 27.023 & 31.002 \\
\hline 37 & 10 & 1.317 & 8.309 & 11.174 & 13.003 & 14.017 & 17.064 & 19.053 & 20.045 & 25.014 & 29.004 \\
\hline 38 & 10 & 3.248 & 4.105 & 11.048 & 13.046 & 14.306 & 15.016 & 17.012 & 18.121 & 21.079 & 27.019 \\
\hline 39 & 10 & 2.089 & 3.199 & 4.099 & 6.365 & 10.102 & 13.090 & 15.042 & 18.003 & 26.007 & 28.005 \\
\hline 40 & 6 & 2.527 & 3.110 & 4.055 & 6.201 & 10.057 & 12.051 & & & & \\
\hline 41 & 10 & 2.088 & 3.195 & 4.099 & 6.354 & 10.106 & 15.093 & 15.046 & 18.004 & 26.009 & 28.007 \\
\hline 42 & 7 & 2.506 & 3.112 & 4.058 & 6.203 & 10.063 & 12.057 & 22.001 & & & \\
\hline 43 & 10 & 2.087 & 3.189 & 4.100 & 6.339 & 10.110 & 13.098 & 15.051 & 18.005 & 26.012 & 28.010 \\
\hline 44 & 6 & 2.190 & 3.445 & 4.280 & 6.059 & 10.013 & 12.012 & & & & \\
\hline 45 & 10 & 9.160 & 11.086 & 14.465 & 17.031 & 19.025 & 20.021 & 23.118 & 27.062 & 31.023 & 35.009 \\
\hline 46 & 7 & 1.820 & 5.062 & 7.025 & 8.005 & 9.082 & 16.005 & 24.001 & & & \\
\hline 47 & 10 & 2.484 & 3.113 & 4.060 & 6.202 & 10.067 & 12.061 & 13.002 & 21.002 & 22.001 & 28.007 \\
\hline 48 & 10 & 2.468 & 3.115 & 4.063 & 6.202 & 10.071 & 12.065 & 13.002 & 21.003 & 22.002 & 28.009 \\
\hline 49 & 10 & 1.647 & 5.047 & 7.017 & B. 059 & 16.105 & 19.012 & 23.051 & 23.044 & 34.011 & 35.008 \\
\hline 51 & 10 & 2.450 & 3.116 & 4.066 & 6.201 & 20.076 & 12.070 & 13.003 & 21.004 & 22.003 & 28.012 \\
\hline
\end{tabular}


Table B-2d. (Continued).

IEVEL NBR

BRANCHING FRACTIONS

$\begin{array}{llrrrrrrrrrr}52 & 10 & 3.171 & 4.086 & 11.056 & 13.055 & 14.256 & 18.143 & 21.112 & 27.056 & 30.034 & 31.029 \\ 53 & 10 & 6.052 & 11.223 & 13.219 & 14.037 & 15.131 & 17.121 & 20.098 & 26.047 & 28.040 & 29.033 \\ 54 & 10 & 2.171 & 3.399 & 4.261 & 6.062 & 10.018 & 12.016 & 21.033 & 22.026 & 28.002 & 30.011 \\ 55 & 10 & 1.584 & 5.050 & 7.020 & 8.064 & 16.115 & 19.016 & 23.063 & 24.055 & 34.018 & 35.014 \\ 56 & 10 & 2.171 & 3.391 & 4.258 & 6.064 & 10.020 & 12.018 & 21.035 & 22.028 & 28.002 & 30.013 \\ 57 & 10 & 6.052 & 11.211 & 13.208 & 14.039 & 15.130 & 17.121 & 20.101 & 26.053 & 28.046 & 29.039 \\ 58 & 10 & 2.423 & 3.119 & 4.070 & 6.200 & 10.082 & 12.076 & 13.004 & 21.005 & 22.004 & 28.017 \\ 59 & 10 & 11.210 & 13.207 & 14.040 & 15.131 & 17.122 & 20.103 & 25.064 & 26.055 & 29.041 & 32.029 \\ 60 & 10 & 2.270 & 3.381 & 4.255 & 6.066 & 10.021 & 12.019 & 21.039 & 22.032 & 28.003 & 30.025 \\ 61 & 10 & 2.086 & 3.166 & 4.100 & 6.274 & 10.118 & 13.109 & 15.071 & 26.031 & 28.027 & 33.017 \\ 62 & 10 & 2.410 & 3.123 & 4.075 & 6.202 & 10.089 & 12.083 & 22.006 & 40.006 & 42.004 & 47.002 \\ \text { end } & & & & & & & & & & & \end{array}$

Table B-2e. File used to convert bandhezd level numbers.

$\begin{array}{llllllllllllllllllllllllll}1 & 1 & 2 & 2 & 3 & 4 & 4 & 10 & 5 & 11 & 6 & 13 & 7 & 22 & 8 & 25 & 9 & 28 & 10 & 29 & 11 & 32 & 12 & 33 & 13 & 34\end{array}$

$\begin{array}{llllllllllllllllllllll}14 & 35 & 15 & 49 & 16 & 50 & 17 & 52 & 18 & 55 & 19 & 56 & 20 & 57 & 21 & 60 & 22 & 67 & 23 & 68 & 24 & 72\end{array}$

$\begin{array}{llllllllllllllllllll}25 & 74 & 26 & 79 & 27 & 83 & 28 & 89 & 29 & 91 & 30 & 95 & 31 & 101 & 32 & 104 & 33 & 105 & 34 & 110\end{array}$

$\begin{array}{llllllllllllllllllll}35 & 118 & 36 & 119 & 37 & 125 & 38 & 128 & 39 & 136 & 40 & 137 & 41 & 146 & 42 & 156 & 43 & 165 & 44 & 166\end{array}$

$\begin{array}{llllllllllllllllllll}45 & 167 & 46 & 169 & 47 & 174 & 48 & 186 & 49 & 195 & 50 & 202 & 51 & 211 & 52 & 215 & 53 & 217 & 54 & 230\end{array}$

$\begin{array}{llllllllllllllll}55 & 234 & 56 & 242 & 57 & 247 & 58 & 249 & 59 & 252 & 60 & 259 & 61 & 272 & 62 & 285\end{array}$ 
Table B-2f. Branching fractions with true level numbers.

LEVEL NBR

BRANCHING FRACTIONS

\begin{tabular}{|c|c|c|c|c|c|c|c|c|c|c|c|}
\hline 4 & 1 & 2.000 & & & & & & & & & \\
\hline 10 & 2 & 2.175 & 4.825 & & & & & & & & \\
\hline 11 & 1 & 1.000 & & & & & & & & & \\
\hline 13 & 2 & 2.981 & 4.019 & & & & & & & & \\
\hline 22 & 2 & 1.842 & 11.158 & & & & & & & & \\
\hline 25 & 1 & 1.000 & & & & & & & & & \\
\hline 28 & 3 & 1.885 & 11.113 & 22.002 & & & & & & & \\
\hline 29 & 4 & 2.830 & 4.064 & 10.013 & 13.093 & & & & & & \\
\hline 32 & 1 & 25.000 & & & & & & & & & \\
\hline 33 & 4 & 2.817 & 4.067 & 10.014 & 13.101 & & & & & & \\
\hline 34 & 1 & 13.000 & & & & & & & & & \\
\hline 35 & 2 & 32.650 & 34.350 & & & & & & & & \\
\hline 49 & 4 & 13.236 & 32.401 & 34.359 & 35.004 & & & & & & \\
\hline 50 & 4 & 1.580 & 11.349 & 22.068 & 25.003 & & & & & & \\
\hline 52 & 4 & 25.013 & 32.514 & 34.466 & 35.006 & & & & & & \\
\hline 55 & 5 & 4.684 & 10.172 & 32.009 & 34.008 & 35.126 & & & & & \\
\hline 56 & 2 & 1.976 & 25.024 & & & & & & & & \\
\hline 57 & 6 & 25.012 & 32.496 & 34.460 & 35.010 & 49.016 & 52.006 & & & & \\
\hline 60 & 4 & 4.666 & 10.304 & 13.028 & 29.001 & & & & & & \\
\hline 67 & 6 & 2.167 & 4.541 & 10.262 & 13.028 & 29.001 & 33.001 & & & & \\
\hline 68 & 4 & 25.255 & 32.062 & 35.680 & 52.002 & & & & & & \\
\hline 72 & 5 & 1.497 & 11.372 & 22.117 & 25.009 & 50.005 & & & & & \\
\hline 74 & 7 & 1.505 & 25.330 & 32.128 & 35.005 & 52.016 & 56.010 & 57.006 & & & \\
\hline 79 & 9 & 13.084 & 29.004 & 32.371 & 34.354 & 35.018 & 49.081 & 52.060 & 55.002 & 57.027 & \\
\hline 83 & 8 & 32.075 & 34.071 & 35.700 & 49.011 & 52.007 & 55.121 & 57.003 & 68.011 & & \\
\hline 89 & 7 & 2.093 & 4.230 & 10.096 & 13.434 & 29.072 & 34.059 & 49.015 & & & \\
\hline 91 & 9 & 25.013 & 32.370 & 34.356 & 35.022 & 49.104 & 52.083 & 55.003 & 57.044 & 74.003 & \\
\hline 95 & 7 & 4.605 & 10.326 & 13.045 & 29.005 & 34.004 & 55.009 & 60.005 & & & \\
\hline 101 & 9 & 32.076 & 34.073 & 35.592 & 49.018 & 52.014 & 55.169 & 57.007 & 68.044 & 83.007 & \\
\hline 104 & 9 & 1.373 & 25.326 & 32.166 & 34.002 & 35.012 & 52.048 & 56.037 & 57.030 & 74.006 & \\
\hline 105 & 10 & 13.066 & 29.005 & 32.321 & 34.311 & 35.024 & 49.112 & 52.093 & 55.005 & 57.058 & 79.005 \\
\hline 110 & 7 & 1.432 & 11.367 & 22.154 & 25.018 & 50.025 & 56.001 & 72.004 & & & \\
\hline 118 & 10 & 25.189 & 32.084 & 35.582 & 50.003 & 52.020 & 56.015 & 57.012 & 68.073 & 74.002 & 83.022 \\
\hline 119 & 10 & 32.076 & 34.073 & 35.517 & 49.023 & 52.019 & 55.187 & 57.011 & 68.070 & 83.0231 & 101.002 \\
\hline 125 & 10 & 1.317 & 25.309 & 32.174 & 34.003 & 35.017 & 52.064 & 56.053 & 57.045 & 74.014 & 91.004 \\
\hline 128 & 10 & 4.248 & 10.105 & 32.048 & 34.046 & 35.306 & 49.016 & 52.013 & 55.121 & 60.079 & 83.019 \\
\hline 136 & 10 & 2.089 & 4.199 & 10.099 & 13.365 & 29.102 & 34.090 & 49.042 & 55.003 & 79.007 & 89.005 \\
\hline 137 & 6 & 2.527 & 4.110 & 10.055 & 13.201 & 29.057 & 33.051 & & & & \\
\hline 146 & 10 & 2.088 & 4.195 & 10.099 & 13.354 & 29.106 & 34.093 & 49.046 & 55.004 & 79.009 & 89.007 \\
\hline 156 & 7 & 2.506 & 4.112 & 10.058 & 13.203 & 29.063 & 33.057 & 67.001 & & & \\
\hline 165 & 10 & 2.087 & 4.189 & 10.100 & 13.339 & 29.110 & 34.098 & 49.051 & 55.005 & 79.012 & 89.010 \\
\hline 166 & 6 & 2.190 & 4.445 & 10.280 & 13.059 & 29.013 & 33.012 & & & & \\
\hline 167 & 10 & 25.160 & 32.086 & 35.465 & 52.031 & 56.025 & 57.021 & 68.118 & 83.0621 & 101.0231 & 118.009 \\
\hline 169 & 7 & 1.820 & 11.062 & 22.025 & 25.005 & 28.082 & 50.005 & 72.001 & & & \\
\hline 174 & 10 & 2.484 & 4.113 & 10.060 & 13.202 & 29.067 & 33.061 & 34.002 & 60.002 & 67.001 & 89.007 \\
\hline 186 & 10 & 2.468 & 4.115 & 10.063 & 13.202 & 29.071 & 33.065 & 34.002 & 60.003 & 67.002 & 89.009 \\
\hline 195 & 10 & 1.647 & 11.047 & 22.017 & 25.059 & 50.105 & 56.012 & 68.051 & 72.0441 & 110.0111 & 118.008 \\
\hline 202 & 10 & 4.488 & 10.312 & 13.069 & 29.019 & 34.016 & 35.003 & 49.008 & 55.043 & 60.033 & 95.009 \\
\hline 211 & 10 & 2.450 & 4.116 & 10.056 & 13.201 & 29.076 & 33.070 & 34.003 & 60.004 & 67.003 & 89.012 \\
\hline
\end{tabular}


Table B-2f. (Continued).

\section{LEVEL NBR BRANCHING ERACTIONS}

\begin{tabular}{rrrrrrrrrrrr}
215 & 10 & 4.171 & 10.086 & 32.056 & 34.055 & 35.256 & 55.143 & 60.112 & 83.056 & 95.034101 .029 \\
217 & 10 & 13.052 & 32.223 & 34.219 & 35.037 & 49.131 & 52.121 & 57.098 & 79.047 & 89.040 & 91.033 \\
230 & 10 & 2.171 & 4.399 & 10.261 & 13.062 & 29.018 & 33.016 & 60.033 & 67.026 & 89.002 & 95.011 \\
234 & 10 & 1.584 & 11.050 & 22.020 & 25.064 & 50.115 & 56.016 & 68.063 & 72.055110 .018118 .014 \\
242 & 10 & 2.171 & 4.391 & 10.258 & 13.064 & 29.020 & 33.018 & 60.035 & 67.028 & 89.002 & 95.013 \\
247 & 10 & 13.052 & 32.211 & 34.208 & 35.039 & 49.130 & 52.121 & 57.101 & 79.053 & 89.046 & 91.039 \\
249 & 10 & 2.423 & 4.119 & 10.070 & 13.200 & 29.082 & 33.076 & 34.004 & 60.005 & 67.004 & 89.017 \\
252 & 10 & 32.210 & 34.207 & 35.040 & 49.131. & 52.122 & 57.103 & 74.064 & 79.055 & 91.041104 .029 \\
259 & 10 & 2.170 & 4.381 & 10.255 & 13.066 & 29.021 & 33.019 & 60.039 & 67.031 & 89.003 & 95.015 \\
272 & 10 & 2.086 & 4.166 & 10.100 & 13.274 & 29.118 & 34.109 & 49.071 & 79.031 & 89.027105 .017 \\
285 & 10 & 2.410 & 4.123 & 10.075 & 13.202 & 29.089 & 33.083 & 67.006137 .006156 .004174 .002 \\
\hline
\end{tabular}


Table B-2h is a portion of the data-analysis table for the multipole transitions that shows the lower 26 out of the 62 band-head levels. The numbering of the parent and daughter levels is the original numbering, before the true level numbers were introduced. Note that a restriction to only dipole transitions would force the $4^{-}$level at $0.658 \mathrm{MeV}$ wo be diagnosed as an isomer, and the 3- level at $0.662 \mathrm{MeV}$ to decay to the above 4- level by an $\mathrm{Ml}$ transition rather than by an E2 transition to the 1- level at $0.390 \mathrm{Mev}$, as shown. Allowing even a small E2 strength can significantly change the relative populations of the ground state and the true isomer at $0.123 \mathrm{MeV}$.

Table B-2h. Portion of the data-analysis output file for ${ }^{176} \mathbf{L u}$.

\section{GAMMA-RAY TRANSITIONS AMONG DISCRETE LEVELS}

Initial Level

N E $\quad$ J $P$

$3.0-1$

Possible Multipoles $B R$ F $/ E G \star *(2 L+1)$ :

$+$

Possible Multipoles $B R E / E G \star \star(2 L+1)$ :

Possible Multipoles $B R E / E G^{\star *}(2 L+1)$ :

Possible Multipoles BR $E / E G \star \star(2 L+1)$ :

Possible Multipoles BR $F / E G^{\star \star}(2 L+1)$ :

$+$

Possible Multipoles BR $F / E G^{\star \star}(2 L+1)$ :

Possible Multipoles $B R E / E G \star(2 L+1)$ :

Possible Multipoles $\mathrm{BR} F / E G^{\star \star}(2 \mathrm{~L}+1)$ :

Possible Multipoles $B R E / E G^{\star} \star(2 L+1)$ :
Einal Level

$\begin{array}{llllll}N & \text { E } & \text { J } & \text { P } & \text { EG } & \text { BR } E\end{array}$

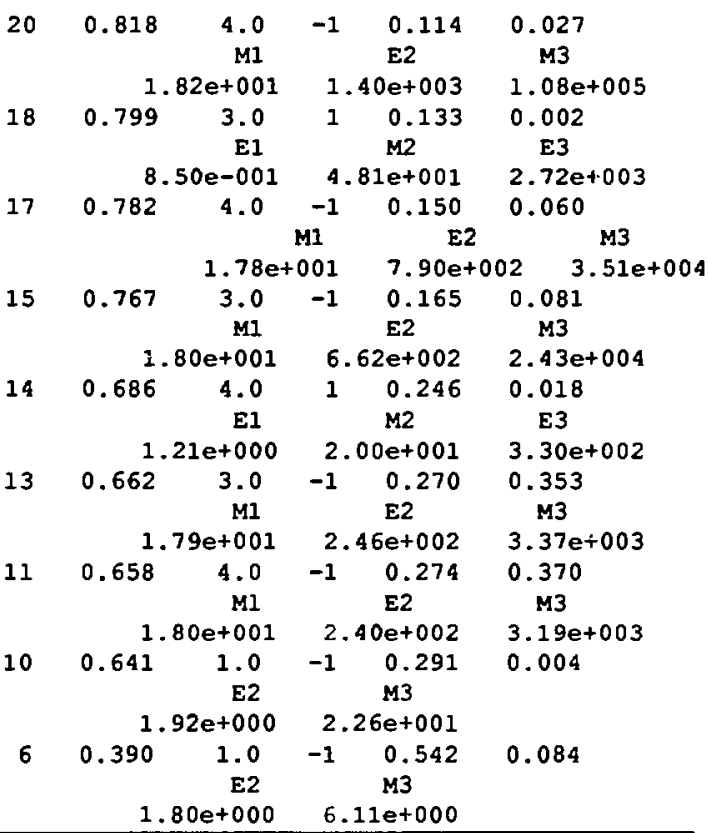


Initial Level

$\begin{array}{llll}\text { N } & \text { E } & \text { J } & \text { P }\end{array}$
Final Irevel

$\begin{array}{llllll}\text { N } & \text { E } & \text { J } & \text { P } & \text { EG } & \text { BR E }\end{array}$

20

$$
\begin{array}{rrr}
0.818 \quad 4.0 & -1 \\
& \mathrm{Ml} &
\end{array}
$$$$
0.089
$$

$\mathrm{E} 2$

$$
8.51 e+000 \quad 1.07 e+003
$$

19

Possible Multipoles $\mathrm{BR} E / E \mathrm{E}^{*} *(2 \mathrm{~L}+1)$ :

$+$

Possible Multipoles BR $E / E G^{\star \star}(2 L+1)$ :

Possible Multipoles BR $E / E G^{\star \star}(2 L+1)$ :

$+$

Possible Multipoles BR $E / E G^{\star \star}(2 L+1)$ :

Possible Multipoles BR $F / E G^{\star \star}(2 L+1)$ :

Possible Multipoles BR $E / E G^{\star \star}(2 L+1)$ :

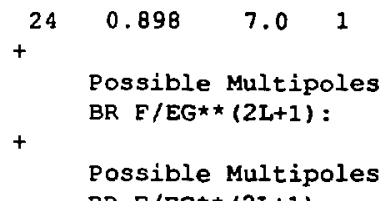
BR $E / E G^{\star \star}(2 L+1)$ :

$$
+
$$

Possible Multipoles BR $F / E G \star \star(2 L+1)$ :

$$
+
$$

Possible Multipoles $\mathrm{BR} F / E G * *(2 \mathrm{~L}+1)$ :

Possible Multipoles $\mathrm{BR} F / E G * *(2 L+1)$ :

17

$$
8.64 e+000 \quad 7.84 e+002
$$

8. $19 \mathrm{e}+000$

$5.24 \mathrm{e}+002$

$$
\text { E1 }
$$

$\mathrm{M} 2$

11

$$
\begin{array}{llll}
0.658 & 4.0 & -1 & 0.249
\end{array}
$$
$\mathrm{MI}$

E2

0. $29 e+000 \quad 1.34 e+002$
$\begin{array}{lllll}8 & 0.566 & 6.0 & -1 & 0.341\end{array}$ M1 E2

$8.32 \mathrm{e}+000 \quad 7.16 \mathrm{e}+001$

$$
\begin{array}{cccc}
0.000 & 7.0 & -1 & 0.907 \\
& \mathrm{E} 2 & & \mathrm{M} 3
\end{array}
$$

8.23e-001 $1.00 e+000$

$$
8.23 e-001 \quad 1.00 e+000
$$

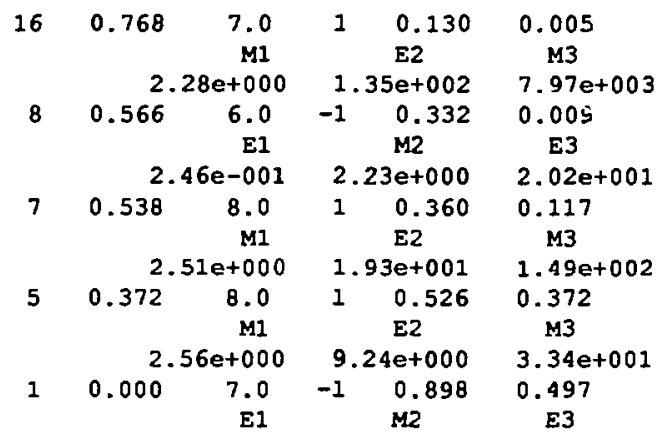

$6.86 e-001 \quad 8.51 e-001$
0.006
M3
1. $36 \mathrm{e}+005$

$$
\begin{array}{ccccc}
0.802 & 6.0 & -1 & 0.105 & 0.010 \\
& \mathrm{MI} & & \mathrm{E} 2 & \mathrm{M} 3
\end{array}
$$

$7.11 e+004$

$$
\begin{array}{ccccc}
0.782 & 4.0 & -1 & 0.125 & 0.016 \\
& \mathrm{M} 1 & & \mathrm{E} 2 & \mathrm{M} 3
\end{array}
$$

$3.36 \mathrm{e}+004$

$$
\begin{array}{lllll}
0.686 & 4.0 & 1 & 0.221 & 0.005
\end{array}
$$

E3

$1.94 \mathrm{e}+002$

0.128

$2.16 \mathrm{e}+003$

0.330 M3

$6.16 \mathrm{e}+002$

0.505

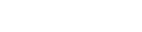




\section{Initial Level}

$\begin{array}{llll}\text { N } & \text { E } & \text { J } & \text { P }\end{array}$

\footnotetext{
$\begin{array}{llll}23 & 0.878 & 5.0 \quad 1\end{array}$

+ Possible Multipoles BR $F / E G^{\star} \star(2 L+1)$ :

+ Possible Multipoles BR F/EG** $(2 L+1)$ :

+ Possible Multipoles $B R F / E G^{\star *}(2 L+1)$ : BR $F / E G * *(2 L+1)$ :
}

$\begin{array}{llllll}17 & 0.782 & 4.0 & -1 & 0.096 & 0.002\end{array}$ E1 $\mathrm{M2} \quad \mathrm{E3}$

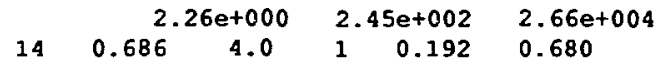 $\mathrm{M1} \quad \mathrm{E2} \quad \mathrm{M3}$

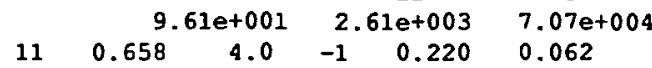

E1 $\mathrm{M2} \quad \mathrm{E} 3$
$\begin{array}{lll}5.82 e+000 & 1.20 e+002 & 2.49 e+003\end{array}$
$\begin{array}{llllll}8 & 0.566 & 6.0 & -1 & 0.312 & 0.255\end{array}$
E1 M2 E3
B. $40 e+000 \quad$ B. $63 e+001 \quad 8.86 e+002$


Initial Level

N E

$\begin{array}{llll}21 & 0.840 & 2.0 & 1\end{array}$

+ Possible Multipoles BR $E / E G \star *(2 L+1)$ :

$+$

Possible Multipoles BR $F / E G^{\star *}(2 L+1)$ :

$+$

Possible Multipoles $B R F / E G * *\{2 L+1)$ :

$+$

Possible Multipoles $\mathrm{BR} F / E G^{\star *}(2 \mathrm{~L}+1)$ :

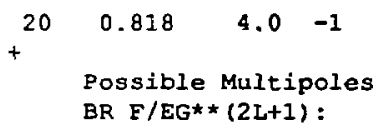

Possible Multipoles BR $E / E G * *(2 L+1)$ :

Possible Multipoles BR $E / E G^{\star *}(2 L+1)$ :

Possible Multipoles $\mathrm{BR} F / \mathrm{EG}^{\star} \star(2 \mathrm{~L}+1)$ :

Possible Multipoles BR $E / E G \star *(2 L+1)$ :

Possible Multipoles BR $E / E G \star \star(2 L+1)$ :

$\begin{array}{llll}19 & 0.802 & 6.0 & -1\end{array}$ $+$

Possible Multipoles BR F/EG** $(2 L+1)$ :

Possible Multipoles BR $E / E G \star *(2 L+1)$ :
Einal Level

$\begin{array}{lllllll}\text { N } & \text { E } & \text { J } & \text { P } & \text { EG } & \text { BR }\end{array}$ $\begin{array}{llllll}17 & 0.782 & 4.0 & -1 & 0.036 & 0.006\end{array}$

$\mathrm{M1} E 2$

$1.29 \mathrm{e}+002 \quad 9.92 \mathrm{e}+004$

$\begin{array}{lllll}15 & 0.767 & 3.0 & -1 & 0.051\end{array}$ M1 E2

$1.21 e+002 \quad 4.64 e+004$

$\begin{array}{lllll}14 & 0.686 & 4.0 & 1 & 0.132\end{array}$ E1

M2

7. $66 \mathrm{e}+007$

0.016

M3

$1.78 \mathrm{e}+007$

0.010

4. $35 \mathrm{e}+000 \quad 2.50 \mathrm{e}+002$ E3

$1.43 e+004$

$\begin{array}{llllll}13 & 0.662 & 3.0 & -1 & 0.156 & 0.460\end{array}$

$$
\text { M1 E2 }
$$

$1.21 \mathrm{e}+002 \quad 4.98 \mathrm{e}+003$

$11 \quad 0.658 \quad 4.0 \quad-1 \quad 0.160$

2. 05 e+005 M1

E2

0.496

M3

$1.21 e+002 \quad 4.73 e+003$

$\begin{array}{lllll}8 & 0.566 & 6.0 & -1 & 0.252\end{array}$

$1.85 \mathrm{e}+005$

0.012

M3

$1.18 \mathrm{~B}+001 \quad 1.8 \mathrm{Ee}+0.02$ $\begin{array}{llllll}8 & 0.56 E & 6.0 & -1 & 0.236 & 0.024\end{array}$ M1 E2

$1.83 e+000 \quad 3.28 e+001$

$\begin{array}{lllll}1 & 0.000 & 7.0 & -1 & 0.802\end{array}$ M1 $\mathrm{E2}$
$\mathrm{M} 3$

5. $89 e+002$

0.976

M3

$1.89 \mathrm{e}+000 \quad 2.94 \mathrm{e}+000 \quad 4.57 \mathrm{e}+000$




\section{Initial Level}

$\begin{array}{llll}\text { N } & \text { E } & \text { J }\end{array}$

$\begin{array}{llll}17 & 0.782 & 4.0 & -1\end{array}$$$
+
$$

Possible Multipoles BR $F / E G \star \star(2 L+1)$ :

Possible Multipoles BR $F / E G * *(2 L+1)$ :

Possible Multipoles BR $F / E G^{\star \star}(2 L+1)$ :

Possible Multipoles $\mathrm{BR} F / E G^{\star \star}(2 I+1)$ :

\section{$\begin{array}{llll}16 & 0.768 & 7.0 & 1\end{array}$}

$+\quad 0.768$ possible Multipoles $\mathrm{BR} E / E G \star \star(2 L+1)$ :

Possible Multipoles BR $F / E G \star \star(2 L+1)$ :

Possible Multipoles BR $F / E G \star *(2 L+1)$ :

Possible Multipoles BR F/EG** $(2 I+1)$ :
Final Level

$\begin{array}{lllllll}\mathbf{N} & \mathbf{E} & \mathbf{J} & \mathbf{P} & \text { EG } & \text { BR } ~\end{array}$

\begin{tabular}{|c|c|c|c|c|c|}
\hline 14 & 0.686 & $\begin{array}{r}4.0 \\
M 1 \\
e+001\end{array}$ & 6. & $\begin{array}{l}0.113 \\
E 2 \\
4 e+003\end{array}$ & $\begin{array}{c}0.126 \\
M 3 \\
5.36 e+005\end{array}$ \\
\hline 13 & 0.662 & 3.0 & -1 & 0.137 & 0.008 \\
\hline & & $\begin{array}{c}E 1 \\
e+000\end{array}$ & & $\frac{122}{6 e+002}$ & $\begin{array}{c}E 3 \\
8.83 e+003\end{array}$ \\
\hline 1 & 0.658 & $\begin{array}{r}4.0 \\
\text { E1 }\end{array}$ & -1 & $\begin{array}{l}0.141 \\
\mathrm{M} 2\end{array}$ & $\begin{array}{c}0.009 \\
\text { E3 }\end{array}$ \\
\hline & & $e+000$ & & $1 e+002$ & B. $12 e+003$ \\
\hline & 0.343 & $\begin{array}{c}1.0 \\
E 2 \\
e+000\end{array}$ & 1 & $\begin{array}{l}0.456 \\
\mathrm{M} 3 \\
\mathrm{ee}+001\end{array}$ & 0.172 \\
\hline & $\begin{array}{r}0.198 \\
\text { B. }\end{array}$ & $\begin{array}{c}1.0 \\
E 2 \\
+000\end{array}$ & & $\begin{array}{l}0.601 \\
M 3 \\
2 e+001\end{array}$ & 0.684 \\
\hline
\end{tabular}
$\begin{array}{llllll}14 & 0.686 & 4.0 & 1 & 0.096 & 0.006\end{array}$ E1 M2 E3
$6.78 \mathrm{e}+000 \quad 7.36 \mathrm{e}+002 \quad 7.98 \mathrm{Be}+004$
$\begin{array}{llllll}13 & 0.662 & 3.0 & -1 & 0.120 & 0.466\end{array}$ M1 E2 M3
$2.70 \mathrm{e}+002 \quad 1.87 \mathrm{e}+004 \quad 1.30 \mathrm{e}+006$
$\begin{array}{llllll}11 & 0.658 & 4.0 & -1 & 0.124 & 0.514\end{array}$ M1 E2 M3
$2.70 e+002 \quad 1.75 e+004 \quad 1.14 e+006$
$\begin{array}{llllll}8 & 0.566 & 6.0 & -1 & 0.216 & 0.013\end{array}$ E2 $\mathrm{M3}$

$2.76 e+001 \quad 5.93 e+002$
$\begin{array}{llllll}8 & 0.566 & 6.0 & -1 & 0.202 & 0.003\end{array}$
E1 $\mathrm{M2}$
E3
$\begin{array}{llllll}7 & 0.538 & 8.0 & 1 & 0.230 & 0.068\end{array}$
$3.64 e-001 \quad 8.92 e+000 \quad 2.19 e+002$
M1 $\mathrm{E2} \quad \mathrm{M3}$
$\begin{array}{llllll}5 & 0.372 & 8.0 & 1 & 0.396 & 0.349\end{array}$
$M 1 \quad$ E2 $\begin{array}{lllll}0.000 & 7.0 & -1 & 0.768 & 0.580\end{array}$
E1 M2 E3
$1.28 e+000 \quad 2.17 e+000 \quad 3.68 e+000$ 
Initial Level

N

E

J $\quad \mathbf{P}$

$\begin{array}{llllll}\text { N } & \text { E } & \text { J } & \text { P } & \text { EG } & \text { BR } F\end{array}$

Final Level

$\begin{array}{lll}15 & 0.767 \quad 3.0 \quad-1 \\ & \text { Possible Multipoles }\end{array}$ BR $F / E G^{\star} \star(2 L+1)$ :

$+$

Possible Multipoles $B R F / E G *(2 I+1)$ :

+ Possible Multipcles $B R F / E G^{\star \star}(2 L+1)$ :

Possible Multipoles $B R E / E G \star *(2 L+1)$ :

$$
\begin{array}{llll}
14 & 0.686 & 4.0 \quad 1
\end{array}
$$

+ Possible Multipoles $B R E / E G \star *(2 L+1)$ :

Possible Multipoles $B R E / E G \star \star(2 L+1)$ :

$$
\begin{array}{llll}
13 & 0.662 & 3.0 & -1
\end{array}
$$

Possible Multipoles

$\begin{array}{llll}12 & 0.659 & 1.0 & -1\end{array}$

+ Possible Multipoles BR $F / E G \star \star(2 L+1)$ :

$$
+
$$

Possible Multipoles BR $F / E G^{\star \star}(2 L+1)$ :

$+$

Possible Multipoles BR $F / E G^{\star \star}(2 L+1)$ :

Possible Multipoles $B R F / E G^{\star \star}(2 L+1)$ :

$$
\begin{array}{llll}
11 & 0.658 & 9.0 & -1
\end{array}
$$

+ Possible Multipoles

$\begin{array}{llllll}14 & 0.686 & 4.0 & 1 & 0.081 & 0.004\end{array}$

$$
\begin{aligned}
& \text { E1 } \quad \text { M2 } \quad \text { E3 } \\
& 7.53 e+000 \quad 1.15 e+003 \quad 1.75 e+005 \\
& \begin{array}{llllll}
13 & 0.662 & 3.0 & -1 & 0.105 & 0.359
\end{array} \\
& \text { M1 E2 M3 } \\
& \begin{array}{llllll}
11 & 0.658 & 4.0 & -1 & 0.109 & 0.401
\end{array} \\
& 3.10 \mathrm{e}+002 \quad 2.81 \mathrm{e}+004 \quad 2.55 \mathrm{e}+006 \\
& M 1 \quad E 2 \quad M 3
\end{aligned}
$$

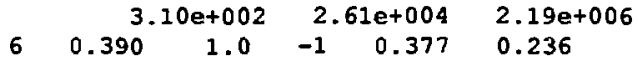

$$
\begin{aligned}
& \begin{array}{cc}
E 2 & \mathrm{M} 3 \\
3.10 \mathrm{e}+001 & 2.18 \mathrm{e}+002
\end{array} \\
& \begin{array}{llllll}
13 & 0.662 & 3.0 & -1 & 0.024 & 0.350
\end{array} \\
& 2.53 e+004 \quad 4.40 e+007 \quad 7.63 e+010 \\
& \begin{array}{llllll}
11 & 0.658 & 4.0 & -1 & 0.028 & 0.650
\end{array} \\
& \text { E1 M2 E3 } \\
& 2.96 e+004 \quad 3.78 \mathrm{e}+007 \quad 5.82 \mathrm{e}+010
\end{aligned}
$$

$\begin{array}{llllll}6 & 0.390 & 1.0 & -1 & 0.272 & 1.000\end{array}$

E2 M3

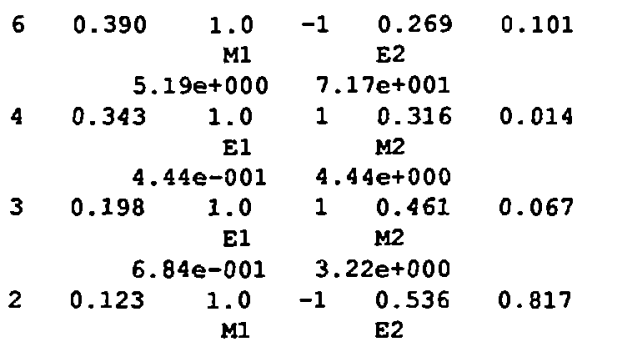

$5.31 e+000 \quad 1.85 e+001$

$\begin{array}{llllll}8 & 0.566 & 6.0 & -1 & 0.092 & 1.000\end{array}$

E2 M3




\section{Initial Level}

$\begin{array}{llll}\text { N } & \text { E } & \text { J } & \text { P }\end{array}$

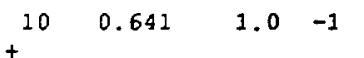

Possible Multipoles $B R$ E $/ E G \star \star(2 L+1)$ :

$+$

Possible Multipoles BR $E / E G \star \star(2 L+1)$ :

$+$

Possible Multipoles $B R F / E G \star(2 L+1)$ :

Possible Multipoles $\mathrm{BR} F / E G^{\star \star}(2 \mathrm{~L}+1)$ :

$$
\begin{array}{llll}
9 & 0.633 & 9.0 & -1
\end{array}
$$

$+$

Possible Multipoles $B R E / E G^{\star} \star(2 L+1)$ :

Possible Multipoles BR $E / E G \star \star(2 L+1)$ :

Possible Multipoles BR $F / E G^{\star \star}(2 L+1)$ :

$$
\begin{array}{lll}
\text { B } & 0.566 & 6.0-1 \\
+ & & \\
& \text { Possible Multipoles }
\end{array}
$$$$
\begin{array}{r}
70.538 \quad 8.01 \\
+\quad 0.04
\end{array}
$$

Possible Multipoles BR $F / E G \star *(2 L+1)$ :

Possible Multipoles BR $F / E G^{\star} \star(2 L+1)$ :

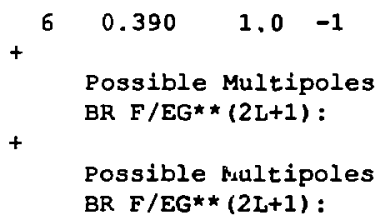

Final Level

$\begin{array}{llllll}\text { N } & \text { E } & \text { J } & \text { P } & \text { EG } & \text { BR F }\end{array}$
6
0.390
$1.0 \quad-1$
0.251
0.093
M1
E2

$$
5.88 e+000 \quad 9.34 e+001
$$

$\begin{array}{lllll}4 & 0.343 & 1.0 & 1 & 0.298\end{array}$

0.013

E1 M2

$4.91 e-001 \quad 5.53 e+000$

$\begin{array}{llllll}3 & 0.198 & 1.0 & 1 & 0.443 & 0.064\end{array}$

E1 1.0

$7.36 e-001 \quad 3.75 e+000$

$\begin{array}{llllll}2 & 0.123 & 1.0 & -1 & 0.518 & 0.830\end{array}$

M1 E2

$5.97 e+000 \quad 2.23 e+001$

$\begin{array}{llllll}7 & 0.538 & 8.0 & 1 & 0.095 & 0.002\end{array}$
E1 $\mathrm{M} 2 \mathrm{E3}$
$2.33 \mathrm{e}+000 \quad 2.58 \mathrm{e}+002 \quad 2.86 \mathrm{e}+004$
$\begin{array}{llllll}5 & 0.372 & 8.0 & 1 & 0.261 & 0.113\end{array}$

E1 $\quad$ M2 $\quad$ E3

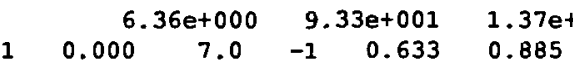

$1.37 e+003$

E2 M3

$8.71 e+000 \quad 2.17 e+001$

$\begin{array}{llllll}1 & 0.000 & 7.0 & -1 & 0.566 & 1.000\end{array}$

M1 E2 M3
$\begin{array}{llllll}5 & 0.372 & 8.0 & 1 & 0.166 & 0.158\end{array}$
$M 1 \quad E 2 \quad M 3$
$3.45 e+001 \quad 1.25 e+003 \quad 4.55 e+004$
$\begin{array}{llllll}1 & 0.000 & 7.0 & -1 & 0.538 & 0.842\end{array}$
E1 $\mathrm{M2} \quad \mathrm{E3}$
$5.41 e+000 \quad 1.87 e+001 \quad 6.45 e+001$




$$
\begin{aligned}
& \text { Initial Level } \\
& \begin{array}{llll}
\text { N } & \text { E } & \text { J } & \text { P }
\end{array} \\
& \begin{array}{llllll}
\text { N } & \text { E } & \text { J } & \text { P } & \text { EG } & \text { BR F }
\end{array} \\
& \begin{array}{ccccccccc}
5 & 0.372 & 8.0 & 1 \\
+ & & & 1 & 0.000 & 7.0 & -1 & 0.372 & 1.000 \\
& \text { Possible Multipoles } & & & \text { E1 } & & \text { M2 } & \text { E3 }
\end{array}
\end{aligned}
$$

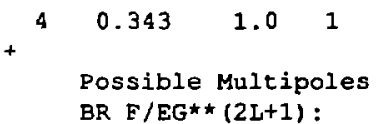

$$
\begin{aligned}
& \begin{array}{llllll}
3 & 0.198 & 1.0 & 1 & 0.145 & 0.825
\end{array} \\
& \text { Possible Multipoles } \\
& \text { BR } E / E G \star \star(2 L+1) \text { : } \\
& \mathrm{M1} \quad \mathrm{E} 2 \\
& 2.71 e+002 \quad 1.29 e+004 \\
& \begin{array}{llllll}
2 & 0.123 & 1.0 & -1 & 0.220 & 0.175
\end{array} \\
& \text { E1 M2 } \\
& 1.64 \mathrm{e}+001 \quad 3.40 \mathrm{e}+002
\end{aligned}
$$

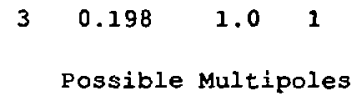

$$
\begin{aligned}
& 20.123 \quad 1.0 \quad-1 \\
& 0.075 \quad 1.000 \\
& \text { E1 M2 } \\
& 2 \quad 0.123 \quad 1.0 \quad-1 \\
& \star \star \star \star * \text { ISOMER } \\
& \begin{array}{llll}
1 & 0.000 & 7.0 & -1
\end{array}
\end{aligned}
$$




\title{
APPENDIX C. Sample NUSTART Structure Output File
}

Table C-1 is a sample of the structure-formated output. This particular sample was generated by using the edit mode on the STAPLUS input file (consisting of the levels given in Table B-2a and the branching fractions given in Table B-2d) and asking for structure output.

Table C-1. Sample NUSTART output.

\author{
RUSTART vers 0.01 .29 \\ Tue Jan $30 \quad 15: 04: 001990$
}

Results for 147602

E. Dep. El cel $=1 . \quad \mathrm{cml}=3.2$ ce2 $=0.32$

$\begin{array}{ccc}\text { E } & J & \text { Branching Eractions / Comments } \\ \text { (keV) } & \text { [Daughter level energy ( } \% \text { branching)] }\end{array}$

$\begin{array}{lll}0 & 7.0- & \\ 123 & 1.0- & \\ 198 & 1.0+ & 123(100.0) \\ 343 & 1.0+ & 123(17.5) 198(82.5) \\ 372 & 8.0+ & 0(100.0) \\ 390 & 1.0- & 123(98.1) 198(1.9) \\ 538 & 8.0+ & 0(84.2) 372(15.8) \\ 566 & 6.0- & 0(100.0) \\ 633 & 9.0- & 0(88.5) 372(11.3) 538(0.2) \\ 641 & 1.0- & 123(83.0) 198(6.4) 343(1.3) 390(9.3) \\ 658 & 4.0-\quad 566(100.0) \\ 659 & 1.0- & 123(81.7) 198(6.7) 343(1.4) 390(10.1) \\ 662 & 3.0- & 390(100.0)\end{array}$




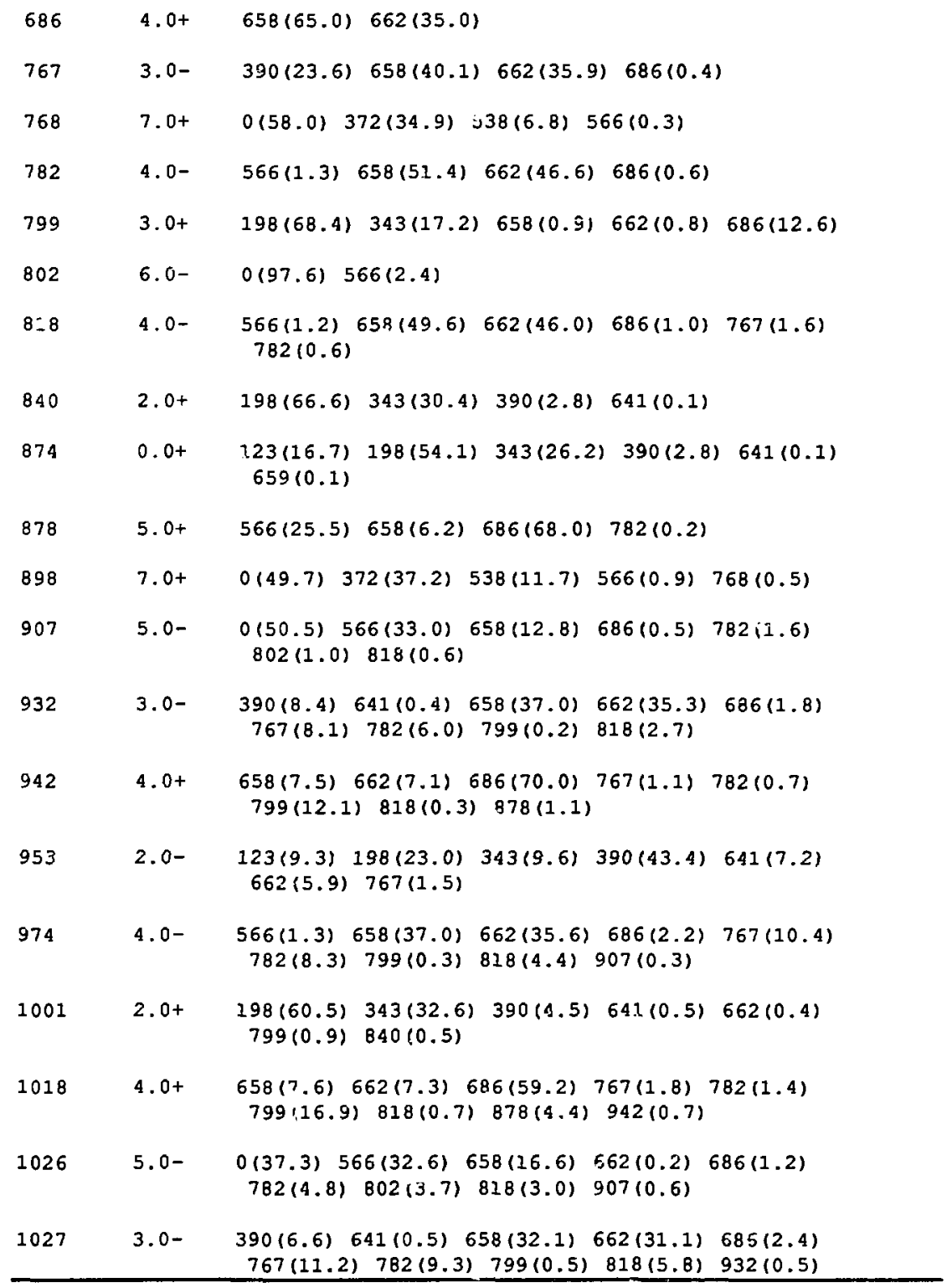




\begin{tabular}{|c|c|c|}
\hline 1046 & $7.0+$ & $\begin{array}{l}0(43.1) 372(36.6) 538(15.3) 566(1.8) 768(2.5) \\
802(0.1) 898(0.4)\end{array}$ \\
\hline 1072 & $5.0+$ & $\begin{array}{cccc}566(18.8) & 658(8.3) & 686(58.0) 768(0.3) 782(2.0) \\
802(1.5) & 818(1.2) & 878(7.2) 907(0.2) 942(2.2)\end{array}$ \\
\hline 1082 & $4.0+$ & $\begin{array}{c}658(7.6) 662(7.3) 686(51.6) 767(2.3) \quad 782(1.9) \\
799(18.6) 818(1.1) 878(7.0) 942(2.3) 1018(0.2)\end{array}$ \\
\hline 1101 & $5.0-$ & $\begin{array}{ccccc}0(31.7) & 566(30.9) & 658(17.4) & 662(0.3) & 686(1.7) \\
782(6.4) & 802(5.3) & 818(4.5) & 907(1.4) & 974(0.4)\end{array}$ \\
\hline 1113 & $3.0+$ & $\begin{array}{rlrll}198(24.7) & 343(10.4) & 558(4.8) & 662(4.6) & 686(30.5) \\
767(1.6) & 782(1.3) & 799(12.0) & 840(7.9) & 942(1.9)\end{array}$ \\
\hline 1131 & $2.0-$ & $\begin{array}{ccccc}123(8.9) & 198(19.8) & 343(9.9) & 390(36.4) & 641(10.1) \\
662(9.0) & 767(4.2) & 799(0.3) & 932(0.7) & 953(0.5)\end{array}$ \\
\hline 1133 & $1.0-$ & $\begin{array}{r}123(52.6) \\
659(5.1)\end{array}$ \\
\hline 1163 & $2.0-$ & $\begin{array}{ccccc}123(8.8) & 198(19.4) & 343(9.9) & 390(35.3) & 641(10.5) \\
662(9.3) & 767(4.5) & 799(0.4) & 932(0.5) & 953(0.7)\end{array}$ \\
\hline 1186 & $1.0-$ & $\begin{aligned} 123(50.6) & 198(11.2) 343(5.8) 390(20.3) 641(6.3) \\
659(5.7) & 874(0.1)\end{aligned}$ \\
\hline 1210 & $2.0-$ & $\begin{array}{ccccc}123(8.7) & 198(18.8) & 343(10.0) & 390(33.8) & 641(10.9) \\
662(9.8) & 767(5.1) & 799(0.5) & 932(1.2) & 953(1.0)\end{array}$ \\
\hline 1211 & $0.0+$ & $\begin{aligned} 127(19.0) & 198(44.5) 343(28.0) 390(5.9) 641(1.3) \\
659(1.2) & \end{aligned}$ \\
\hline 1214 & $5.0+$ & $\begin{array}{rllll}566(16.0) & 658(8.6) & 686(46.5) & 782(3.1) & 802(2.5) \\
818(2.1) & 878(11.8) & 942(6.2) & 1018(2.3) & 1072(0.9)\end{array}$ \\
\hline 1218 & $8.0-$ & $\begin{array}{l}0(82.0) 372(6.2) 538(2.5) 566(0.5) 633(8.2) \\
768(0.5) 898(0.1)\end{array}$ \\
\hline 1231 & $1.0-$ & $\begin{aligned} 123(48.4) & 198(11.3) 343(6.0) 390(20.2) 641(6.7) \\
659(6.1) & 662(0.2) 840(0.2) 874(0.1) 953(0.7)\end{aligned}$ \\
\hline 1273 & $1.0-$ & $\begin{array}{rllll}123(46.8) & 198(11.5) & 343(6.3) & 390(20.2) & 641(7.1) \\
659(6.5) & 662(0.2) & 840(0.3) & 874(0.2) & 953(0.9)\end{array}$ \\
\hline 1292 & $6.0+$ & $\begin{array}{l}0(64.6) 372(4.7) 538(1.7) 566(5.9) 768(10.4) \\
802(1.2) 878(5.1) 898(4.4) 1046(1.1) \quad 1072(0.8)\end{array}$ \\
\hline 1307 & $2.0+$ & $\begin{array}{rrrrr}198(48.8) & 343(31.2) & 390(6.9) & 641(1.9) & 662(1.6) \\
686(0.3) & 767(0.8) & 799(4.3) & 840(3.3) & 1001(0.9) \\
\end{array}$ \\
\hline
\end{tabular}




\begin{tabular}{|c|c|c|}
\hline 1324 & $1.0-$ & $\begin{array}{ccccc}123(44.9) & 198(11.5) 343(6.6) & 390(20.0) 641(7.6) \\
659(7.0) & 662(0.3) & 840(0.4) 874(0.3) \quad 953(1.2)\end{array}$ \\
\hline 1339 & $3.0+$ & $\begin{array}{c}198(17.1) \quad 343(8.6) \quad 658(5.6) 662(5.5) \quad 686(25.6) \\
799(14.3) \quad 840(11.2) \quad 942(5.6) \quad 1001(3.4) \quad 1018(2.9)\end{array}$ \\
\hline 1342 & $3.0-$ & $\begin{array}{ccccc}390(5.2) & 658(22.2) & 662(21.8) & 686(3.7) & 767(13.0) \\
782(12.0) & 818(9.8) & 932(4.7) & 953(4.0) & 974(3.3)\end{array}$ \\
\hline 1367 & $1.0+$ & $\begin{array}{c}123(17.1) 198(39.9) 343(26.1) 390(6.2) 641(1.8) \\
659(1.6) 840(3.3) 874(2.6) 953(0.2) 1001(1.1)\end{array}$ \\
\hline 1379 & $6.0+$ & $\begin{array}{l}0(58.4) 372(5.0) 538(2.0) 566(6.4) 768(11.5) \\
802(1.6) 878(6.3) 898(5.5) 1046(1.8) 1072(1.4)\end{array}$ \\
\hline 1397 & $1.0+$ & $\begin{array}{r}123(17.1) 198(39.1) 343(25.8) 390(6.4) 641(2.0) \\
659(1.8) 840(3.5) 874(2.8) 953(0.2) 1001(1.3)\end{array}$ \\
\hline 1410 & $3.0-$ & $\begin{array}{ccccc}390(5.2) & 658(21.1) & 662(20.8) & 686(3.9) & 767(13.0) \\
782(12.1) & 18(10.1) & 932(5.3) & 953(4.6) & 974(3.9)\end{array}$ \\
\hline 1412 & $1.0-$ & $\begin{array}{ccccc}123(42.3) & 198(11.9) & 343(7.0) & 390(20.0) 641(8.2) \\
659(7.6) & 662(0.4) & 840(0.5) 874(0.4) & 953(1.7)\end{array}$ \\
\hline 1432 & $4.0-$ & $\begin{array}{ccccc}658(20.9) & 662(20.6) & 686(4.0) & 767(13.0) & 782(12.1) \\
818(10.2) & 907(6.3) & 932(5.4) & 974(4.1) & 1026(2.9)\end{array}$ \\
\hline 1440 & $1.0+$ & 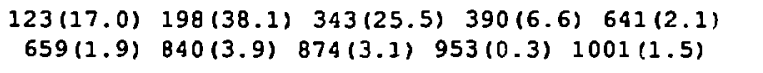 \\
\hline 1468 & $2.0-$ & $\begin{array}{c}123(8.6) 198(16.6) 343(100) 390(27.4) 641(11.8) \\
662(10.9) 767(7.1) 932(3.1) 953(2.7) 1027(1.7)\end{array}$ \\
\hline 1489 & $1.0-$ & $\begin{array}{rlrll}123(41.0) & 198(12.3) & 343(7.5) & 390(20.2) & 641(8.9) \\
659(8.3) & 874(0.6) & 1133(0.6) & 1186(0.4) & 1231(0.2)\end{array}$ \\
\hline
\end{tabular}




\section{Appendix D. Error Messages}

When NUSTART encounters an error, the program clears the screen and writes the error message to the screen. It also writes the error message to the data-analysis file. The program pauses until you hit a key to force it to continue. When the error message is only a warning, the program will continue and finish the current process. Fatal errors, hourrer, will cause the program to terminate the current process and return to the main men:s.

Table D-1. NUSTART error messages.

\begin{tabular}{|c|c|}
\hline Error & Comment \\
\hline $\begin{array}{l}\text { 1 Error on reading levels; } \\
\text { possible missing data. }\end{array}$ & $\begin{array}{l}\text { There was a problem reading the } \\
\text { STAPLUS format input deck. Check to verify } \\
\text { that the level information is correct. }\end{array}$ \\
\hline $\begin{array}{l}12 \text { Elror on reading levels: } \\
\text { too few levels were read. }\end{array}$ & $\begin{array}{l}\text { The first card of the STAPLUS file declared } \\
\text { that there were "N" levels in the deck, but } \\
\text { NUSTART encountered an END-OF-FILE } \\
\text { before "N" levels were read. Correct your } \\
\text { input deck. }\end{array}$ \\
\hline $\begin{array}{l}\text { \$ Level energies are out of } \\
\text { sequence. }\end{array}$ & $\begin{array}{l}\text { The level energies must be in numeric order from } \\
\text { low to high. The level energies that are out of } \\
\text { order are lisied in the data-analysis file. Corr } \\
\text { your deck }\end{array}$ \\
\hline $\begin{array}{l}\text { *4 Unexpected END-OF-LINE in } \\
\text { reading branching information. } \\
\text { Check data. }\end{array}$ & $\begin{array}{l}\text { This emor should never occur. If it does occur, } \\
\text { it mpans that the the code could not read the } \\
\text { end_of_line delimiter and there is something } \\
\text { wrong with your data. }\end{array}$ \\
\hline \multicolumn{2}{|l|}{$\begin{array}{l}\text { \#5 Attempt to read too many } \\
\text { branching fractions. Check } \\
\text { the format of the deck. }\end{array}$} \\
\hline $\begin{array}{l}16 \text { Ground state level card } \\
\text { was read. Check the format } \\
\text { of the deck }\end{array}$ & \\
\hline \multicolumn{2}{|l|}{$\begin{array}{l}17 \text { Last branching fraction } \\
\text { level number exceeds the } \\
\text { number given on card } 1 \text { of the } \\
\text { input. Check the input deck. }\end{array}$} \\
\hline $\begin{array}{l}\text { \#8 Number of levels null or } \\
\text { equal zero. }\end{array}$ & $\begin{array}{l}\text { The number of levels given on card } 1 \text { of the } \\
\text { STAPLUS format input deck is a zero or a } \\
\text { negative number. Check the input deck. }\end{array}$ \\
\hline
\end{tabular}


Table D-1 continued.

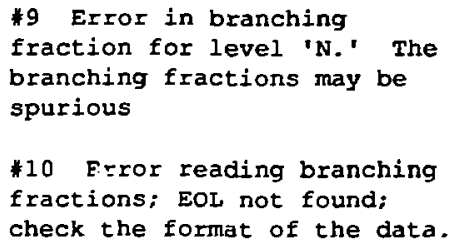

\#11 Could not create output file; please retry.

*12 Could not create DATA ANALYSIS file; terminating.

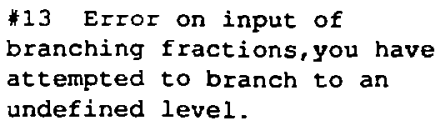

This error occurs during the normalization of the input data. Check your input deck.

This error should never occur. If it does occur, it means that the the code could not read the end_of_line delimiter and there is something wrong with your data.

Check to be sure that you have enough disk space to create a file, or that the path you gave in the file name is a valid path.

You probably do not have enough disk space to create another file, or the path you gave for the file name is not valid.
Check your input deck, the END card appears to he missing. 
Table D-1 continued.

*20 Unexpected END_OF_FILE

The header card stated there were more

while reading branching fractions

levels than exist in the data set. The

data. Data will be used without

branching fractions.

program continued so that the rest of the data could be analyzed. Correct your data set. 